\title{
On the elemental abundance and isotopic mixture of mercury in HgMn stars
}

\author{
L. Dolk ${ }^{1}$, G. M. Wahlgren ${ }^{1}$, and S. Hubrig ${ }^{2}$ \\ 1 Atomic Astrophysics, Lund Observatory, Lund University, Box 43, 22100, Lund, Sweden \\ 2 European Southern Observatory, Casilla 19001, Santiago 19, Chile
}

Received 2 July 2002 / Accepted 13 February 2003

\begin{abstract}
Optical region spectra of $31 \mathrm{HgMn}$ stars have been studied for the abundance and isotope mixture of mercury. In the course of the investigation the lines $\mathrm{Hg}_{\mathrm{I}} \lambda 4358$ and $\mathrm{Hg}$ II $\lambda \lambda 3984,6149$ have been studied, with abundances established for all three lines in several HgMn stars. The mercury isotope mixture has been determined from high resolution spectra of the $\lambda 3984$ line. Possible signs of an ionization anomaly have been detected by the comparison of the abundance derived from the $\mathrm{Hg}$ I line and the $\mathrm{Hg}$ II lines in seven of the observed $\mathrm{HgMn}$ stars. A possible correlation of the mercury abundance with $T_{\text {eff }}$ has been detected. Possible signs of a weak anticorrelation of the manganese and mercury abundance in HgMn stars have been found, which could be interpreted as a sign of inhomogeneous surface distribution of these elements. For a number of the HgMn stars in this study the mercury abundance and isotope mixture are reported for the first time.
\end{abstract}

Key words. stars: abundances - stars: chemically peculiar

\section{Introduction}

The presence of mercury in $\mathrm{HgMn}$ stars has been studied ever since Bidelman (1962a,b) identified the $\lambda 3984 \AA$ feature as due to $\mathrm{Hg}$ II. The abundance of mercury, as well as its isotopic composition, has been reported for a number of $\mathrm{HgMn}$ stars from optical (e.g., Cowley \& Aikman 1975; White et al. 1976; Heacox 1979; Smith 1997; Woolf \& Lambert 1999a) and ultraviolet (Leckrone 1984; Smith 1997; Proffitt et al. 1999) spectra.

It is generally believed that the abundance and isotopic anomalies observed for various elements in $\mathrm{HgMn}$ stars are the result of diffusion processes at work in their atmospheres. The diffusion hypothesis, advanced by Michaud (1970), suggests that the abundance anomalies observed in chemically peculiar (CP) stars are the result of an interplay between the radiative force and gravity. Michaud et al. (1974) described several scenarios where diffusion could produce the observed abundance and isotopic anomalies of mercury. However, Proffitt et al. (1999) reported on calculations for which the mercury abundance in the HgMn star $\chi$ Lupi could not be explained by diffusion alone. Similarly, isotopic anomalies have been observed in certain HgMn stars (Woolf \& Lambert 1999a) that are difficult to explain by diffusion theory alone.

The theory of light induced drift (LID) was put forward (Atutov \& Shalagin 1988) as a possible explanation for abundance anomalies in CP stars. LID occurs when there is an asymmetric light flux across the Doppler-broadened

Send offprint requests to: L. Dolk, e-mail: linus.dolk@astro.lu.se absorption profile of an atomic or ionic line. The asymmetry can arise from the presence of another spectral feature in the line wing, or from a local slope in the continuum. The asymmetric light flux causes the absorbing particles of the element to become selectively excited based on their velocities. For example, if the light flux is higher on the blue side of the line profile, then the particles moving in the direction of light propagation are excited more efficiently. The light flux can thus induce a flow of excited particles in one direction while unexcited particles flow in the opposite direction. Since the collisional cross sections typically are larger for excited than unexcited atoms on account of their larger effective radius, the flow of excited particles is decelerated more efficiently by collisions with the surrounding gas than the unexcited particles. This results in a net flux of absorbing particles in the direction specified by the flux of the less excited atoms. LID can only occur for spectral lines that have upper and lower quantum states with different collisional cross sections. Therefore, the LID introduced particle flux is largest for atomic transitions between low and high energy levels on account of these quantum states typically having noticeably different collisional cross section. Nasyrov \& Shalagin (1993) calculated that LID can be a larger contributor for elemental separation than radiative diffusion. Recently, calculations by Aret \& Sapar (2002) indicate that the isotopic anomalies observed in $\mathrm{HgMn}$ stars can be explained by LID, and that a temporal enhancement of heavy isotopes can be expected on evolutionary timescales.

Few spectral lines of mercury have been incorporated into previous analyses. Efforts have primarily been 
concentrated on the $\mathrm{Hg}_{\text {II }} \lambda 3984$ line, with the $\mathrm{Hg}_{\text {II }} \lambda 1942$ and the $\mathrm{Hg}_{\mathrm{I}} \lambda 4358$ lines being studied to a lesser extent. In the studies by White et al. (1976), Smith (1997), and Woolf \& Lambert (1999a), a relatively large number of HgMn stars were studied for the presence, abundance and isotope mixture of mercury. These optical studies concentrated primarily on the behaviour of the $\mathrm{Hg}$ II $\lambda 3984$ line, but the latter two studied the $\mathrm{Hg}_{\mathrm{I}} \lambda 4358$ line in selected stars. Smith (1997) also included a low-resolution study of $\mathrm{Hg}$ II $\lambda 1942$ using data from the International Ultraviolet Explorer (IUE) satellite. High-resolution ultraviolet studies of additional mercury lines, including $\mathrm{Hg}$ III lines, have been made for few $\mathrm{HgMn}$ stars (Proffitt et al. 1999; Wahlgren et al. 1995).

Further insights into the nature of $\mathrm{HgMn}$ star atmospheres would be benefitted by the study of additional spectral features. For instance, no large-scale study has made use of the optical line $\mathrm{Hg}$ II 26149 . The line has been investigated in the $\mathrm{HgMn}$ star $\mu$ Lep by Takada-Hidai \& Jugaku (1992) and in a small sample of HgMn stars by Hubrig et al. (1999a). This line is observed to be significantly weaker than the $\lambda 3984$ feature, due to a combination of weaker oscillator strength and higher excitation energy, but it is strong enough to be observed in the $\mathrm{HgMn}$ stars provided that the enhancement of mercury is more than 4 dex.

In the present analysis the $\mathrm{Hg}_{\text {I }} \lambda 4358$ line and the $\mathrm{Hg}$ II $\lambda 3984$ and $\lambda 6149$ lines have been studied at high-resolution. The motivation for the present study was to make a comparison of the abundance derived from the three mercury lines to search for possible ionization and excitation anomalies and to investigate the $\mathrm{Hg}$ II $\lambda 3984$ line in a number of sharp-lined HgMn stars to look for trends in the isotopic composition. There was also an interest in studying the $\mathrm{Hg}$ II $\lambda 3984$ line in a sample of broader-lined HgMn stars lacking such investigations.

There is much observational evidence that magnetic CP stars have elements distributed in patches on their stellar surface. In many of these stars the inhomogeneous distribution causes derived abundances to vary noticeably during the rotational period of the star. Recent evidence suggests that there is an inhomogeneous surface distribution of mercury for the HgMn star $\alpha$ And (Wahlgren et al. 2001; Adelman et al. 2002). If the existence of patches is a common occurrence in $\mathrm{HgMn}$ stars this would violate one of the key underlying assumptions of the abundance analysis, that the atmosphere consists of homogeneous layers. In this paper we have also investigated the possibility that the elements mercury and manganese are distributed at different locations on the stellar surface by looking for an anti-correlation of their abundances in HgMn stars, and comparing multiple spectra, where they exist.

\section{Observational data}

The spectral data comprising this study were mainly obtained with instruments located at three observatories as part of different studies undertaken in recent years: the SOFIN echelle spectrograph at the Nordic Optical Telescope (NOT), the ESO Multi-Mode Instrument (EMMI) spectrograph at the New Technology Telescope (NTT), and the Coudé Echelle Spectrometer (CES) at the ESO CAT telescope. For one star,
AV Scl, the analysis was made utilizing data from the UVVisual Echelle Spectrograph (UVES) of the ESO Very Large Telescope (VLT).

The NOT is a $2.56 \mathrm{~m}$ telescope located at the Roque de los Muchachos Observatory on the island of La Palma. The spectra obtained for the present project were taken with cameras 1 and 2 of the SOFIN echelle spectrograph (Tuominen 1992; Ilyin 2000), with signal-to-noise $(S / N)$ ratios mainly above 100 and resolving powers of approximately $R=170000$ and 80000 , respectively.

A substantial number of the spectra were obtained at the ESO 1.4 m CAT telescope utilizing the CES Very Long Camera equipped with CCD Loral \#38. The data taken with the CAT were obtained as part of several observing runs during the period 1995 to 1998 , and were reduced in a normal manner using MIDAS software. The data quality is quite good, as defined by both high resolving power $(R \simeq 100000-135000)$ and $S / N>200$.

Spectra taken with the EMMI spectrograph at the $3.5 \mathrm{~m}$ NTT telescope cover a large wavelength interval (4070 $6740 \AA$ ) at a resolving power of $R \sim 80000$ and $S / N \sim 100$.

The spectrum of AV Scl was taken with the UVES spectrograph at the $8.0 \mathrm{~m}$ VLT. The UVES is a two-arm crossdispersed echelle spectrograph used for observations in the near-ultraviolet and optical spectral regions. The instrumental resolving power used for our spectra were $R \sim 80000$ in the blue and $R \sim 110000$ in the red region. The $S / N$ ratio was greater than 100 .

There is a different amount of data for each of the observed HgMn stars, with some of the stars observed in a small spectral region utilizing one spectrograph, while other stars having been observed several times with more than one instrument in multiple spectral regions. In total, data have been collected for $31 \mathrm{HgMn}$ stars, over half of which have observations for more than one mercury line. An overview of the observational data is presented in Table 1.

\section{Analysis}

\subsection{Stellar parameters}

The stars discussed in this study are mostly sharp-lined $\left(v \sin i<10 \mathrm{~km} \mathrm{~s}^{-1}\right.$ ) HgMn stars, which enables us to study the abundance of individual isotopes of the $\mathrm{Hg}$ II $\lambda 3984$ line. HgMn stars with larger $v \sin i$ are also presented for cases where no mercury abundance had previously been established. Many of the stars chosen for this study are discussed in Dolk et al. (2002). For stars not included in Dolk et al. the effective temperature and surface gravity were calculated from an algorithm of R. Napiwotski (1993; private communication), using the uvby photometric data of Hauck \& Mermilliod (1980). The effective temperatures and surface gravities are presented in Table 1.

More than half of the program stars are spectroscopic binaries, and in cases where both components are known their synthetic spectra were weighted by the estimated luminosity ratio at the key wavelengths and coadded prior to convolution with the instrumental profile. For double-lined spectroscopic binaries (SB2) observed in the study by Dolk et al., 
the same luminosity ratio was utilized in this study. For the remaining SB2 systems the effect of binarity was established by observations of secondary spectral lines, synthetic spectrum calculations, and, in some cases, previous observations. For the stars 41 Eri and HR 7694 photometric data from Hauck \& Mermilliod (1980) were combined with observed Fe I and $\mathrm{Fe}$ II line strengths and noted mass ratio (Guthrie 1986) to yield a luminosity ratio. For HD 1909, HR 6520 and HD 124740 lines from the secondary were noted and the parameters of the secondary and a luminosity ratio were calculated by iterative synthetic calculations. Stars noted as single-line binaries (SB1) in Table 1 were treated as single stars.

\subsection{Abundance analysis and line data}

Elemental abundances and isotopic mixtures were determined by comparing the observed spectra to synthetic spectra generated with the SYNTHE program (Kurucz \& Avrett 1981; Kurucz 1993) and model atmospheres generated with the ATLAS9 code. Atomic line data were taken from the lists of Kurucz (1993) with the exception of the mercury lines and other lines in the observed regions for which improved atomic data were available. The abundance and isotopic mixture of mercury for each star were determined by changing these parameters until the synthetic spectrum matched the observed spectrum. The projected equatorial rotational velocity, $v \sin i$, was determined for each star from line profile fitting of Fe II lines, with lines from other elements being used as confirmation.

In the present paper the optical region mercury lines Hg I $\lambda 4358, H g$ II $\lambda \lambda 3984,6149$ have been studied, and the wavelength and oscillator strength of the hyperfine and isotopic components for each transition are presented in Table 2 for the case of a terrestrial isotopic mixture (Anders \& Grevesse 1989). The wavelengths for the $\lambda 4358$ and the $\lambda 3984$ lines were taken from Wahlgren et al. (2000), while the oscillator strengths are experimental values adopted from Benck et al. (1989) for $\mathrm{Hg}_{\text {I }} \lambda 4358(\log g f=+0.34)$ and Dworetsky (1980) for $\mathrm{Hg}$ II $\lambda 3984(\log g f=-1.73)$. Note should be made that the $\log g f$ value of the $\mathrm{Hg}$ II $\lambda 3984$ line adopted here is $0.2 \mathrm{dex}$ lower than the theoretically calculated values of Proffitt et al. (1999) $(\log g f=-1.529)$ and Sansonetti \& Reader (2001) $(\log g f=-1.51)$. In our investigation the experimentally determined $g f$ value was chosen since its experimental nature allows for an uncertainty to be attached, while the theoretically calculated value is of indeterminable uncertainty. The $\lambda 3984$ line is in principle a forbidden line since it involves a twoelectron jump, and the fact that it can be observed at all is due to the configuration interaction of the levels involved in the transition. The difficulty in calculating the exact admixture of the involved levels results in a large uncertainty of the calculated $g f$ value. Sansonetti \& Reader noted that the laboratory intensity of the $\lambda 3984$ feature is inconsistent with their theoretical $g f$ value. By combining a theoretically calculated branching fraction of the upper level with its lifetime measured by Pinnington et al. (1988), they derive a $\log g f$ value of -1.82 , in agreement with the experimental value of Dworetsky to within the latter's error estimate of 0.2 dex.

The absolute wavelength of the $\mathrm{Hg}$ II $\lambda 6149$ line was set by measurement of the ${ }^{198} \mathrm{Hg}$ isotope (Reader \& Sansonetti 1986). Wavelengths for the even isotope components ${ }^{200} \mathrm{Hg},{ }^{202} \mathrm{Hg}$ and ${ }^{204} \mathrm{Hg}$ were determined relative to the ${ }^{198} \mathrm{Hg}$ wavelength from the measurements of Murakawa (1932), while the wavelengths of the hfs components of the odd isotopes are based upon center-of-gravity estimates from Reader \& Sansonetti (private communication) and the theoretically determined magnetic hfs constant, A, of Brage et al. (1999) for the lower energy level. The hfs constant $A$ for the upper energy level is unknown but has been set to zero since it is anticipated to be small due to the absence of an $s$ valence electron in its configuration. The total $g f$ value of the $\lambda 6149$ line $(\log g f=0.328)$ is taken from the relativistic calculation of Migdalek (1976).

\subsection{Microturbulent velocity}

It is often debated whether or not the microturbulent velocity has any real physical meaning, or if it is just used as a convenient way of explaining observed line broadening effects not otherwise explainable by classical atmosphere modelling techniques. Observations of the solar surface and hydrodynamical models of other stars indicate that there are quasi-random velocity fields in the gas of stellar photospheres. Since no detailed knowledge of these velocity fields exists, the usage of a microturbulence parameter becomes necessary (Cowley 1996). For solar-type stars three-dimensional modelling techniques replace the application of turbulent velocities with a more formal evaluation of the hydrodynamics as a function of atmospheric depth (Asplund et al. 1999).

In the computation of synthetic spectra in the onedimensional approach, the microturbulence is used in fitting line profiles, and is often needed to achieve a good fit. The determination of the microturbulence is usually made by adjusting the microturbulence parameter, $\xi_{\mathrm{t}}$, until there is no systematic difference in the abundances determined from weak and intermediate-strong lines of an atom/ion. For this reason its value is influenced by systematic errors in the oscillator strengths as well as uncertainties in the abundance determinations. Also, typical application of the method assumes that all elements are subjected to the same microturbulence and that its value is constant with atmospheric depth. For HgMn stars the $\xi_{\mathrm{t}}$ parameter is typically found to have small values from both the traditional method of balancing line strength as a function of excitation energy and line profile analysis. Landstreet (1998, 2003) analysed nearly 20 late-B and A type main sequence stars by line profile analysis and finds $\xi_{\mathrm{t}}<1 \mathrm{~km} \mathrm{~s}^{-1}$ for $T_{\text {eff }}>10000 \mathrm{~K}$ but values of up to $5 \mathrm{~km} \mathrm{~s}^{-1}$ for the A stars.

During the early stages of our study it was noted that the three mercury lines behave differently as $\xi_{\mathrm{t}}$ is changed. The effect of $\xi_{t}$ mainly depends on the strength of the line, with stronger lines being more affected. For this reason the stronger $\mathrm{Hg}$ II $\lambda 3984$ line is more sensitive to a change in the $\xi_{\mathrm{t}}$ while the weaker $\mathrm{Hg}$ I $\lambda 4358$ is only marginally affected and $\mathrm{Hg}$ II $\lambda 6149$ is virtually unaffected. Two sets of synthetic spectra were 
Table 1. Parameters of the HgMn stars and observational data.

\begin{tabular}{|c|c|c|c|c|c|c|c|c|c|c|}
\hline \multirow[t]{2}{*}{ HD } & & \multirow[t]{2}{*}{ Name } & \multirow[t]{2}{*}{ Bin. } & \multirow{2}{*}{$\begin{array}{l}T_{\text {eff }} \\
(\mathrm{K})\end{array}$} & \multirow{2}{*}{$\begin{array}{l}\log g \\
(\operatorname{cgs})\end{array}$} & \multirow[t]{2}{*}{$L_{A} / L_{B}{ }^{a}$} & \multirow{2}{*}{$\begin{array}{l}v \sin i \\
\mathrm{~km} \mathrm{~s}^{-1}\end{array}$} & \multicolumn{3}{|c|}{ Observations $^{b}$} \\
\hline & & & & & & & & $\lambda 3984$ & $\lambda 4358$ & $\lambda 6149$ \\
\hline \multirow[t]{2}{*}{1909} & $\mathrm{~A}$ & AV Scl & SB2 & 12400 & 4.00 & 12 & 12 & CAT(2), VLT(2) & VLT(2) & VLT \\
\hline & $\mathrm{B}$ & & & 9000 & 4.00 & & 12 & & & \\
\hline 7374 & & 87 Psc & & 13126 & 4.02 & & 21 & CAT & & \\
\hline 11753 & & $\phi$ Phe & & 10612 & 3.79 & & 14 & CAT(4) & CAT & \\
\hline 19400 & & $\theta$ Hyi & & 14106 & 3.81 & & 36 & CAT & & \\
\hline \multirow{2}{*}{27376} & A & 41 Eri & SB2 & 12750 & 4.18 & 1.25 & 12 & CAT & CAT & \\
\hline & B & & & 12250 & 4.10 & & 12 & & & \\
\hline 33904 & & $\mu$ Lep & & 12750 & 3.77 & & 16.5 & CAT(7), NOT(4) & CAT, NOT(4) & CAT \\
\hline \multirow{2}{*}{35548} & A & HR 1800 & SB2 & 11088 & 3.79 & 4.3 & 2 & CAT(2) & & CAT, NTT \\
\hline & B & & & 8500 & 4.00 & & 50: & & & \\
\hline 71066 & & HR 3302 & & 12010 & 3.95 & & 3 & CAT(2) & & CAT \\
\hline 77350 & & $v \mathrm{Cnc}$ & SB1 & 10375 & 3.50 & & 19 & NOT & NOT & NOT(2) \\
\hline \multirow[t]{2}{*}{78316} & A & $\kappa \mathrm{Cnc}$ & SB2 & 13470 & 3.81 & 11.5 & 6.5 & CAT & NTT & CAT(2), NTT \\
\hline & B & & & 8500 & 4.00 & & 40 & & & \\
\hline \multirow[t]{2}{*}{89822} & A & HR 4072 & SB2 & 10900 & 4.07 & 3.2 & 3.5 & NOT(2) & NOT(2) & NOT \\
\hline & B & & & 8900 & 4.20 & & 5.5 & & & \\
\hline 90264 & & HR 4089 & SB1 & 15126 & 4.16 & & 7 & CAT & & \\
\hline 101189 & & HR 4487 & & 11020 & 3.92 & & 18 & CAT & & \\
\hline \multirow[t]{2}{*}{124740} & A & & SB2 & 10350 & 4.00 & 8 & 2 & CAT(2) & CAT & CAT(2) \\
\hline & B & & & 8000 & 4.00 & & 5 & & & \\
\hline \multirow[t]{2}{*}{141556} & A & $\chi$ Lup & SB2 & 10608 & 3.98 & 3.96 & 2.1 & CAT(7), NOT & NOT, NTT & CAT(2), NTT \\
\hline & B & & & 9200 & 4.20 & & 2 & & & \\
\hline \multirow[t]{2}{*}{143807} & A & $\iota \mathrm{CrB}$ & SB2 & 11250 & 3.65 & 4.0 & 2 & NOT & NOT & NOT, CAT \\
\hline & B & & & 9250 & 4.00 & & 3 & & & \\
\hline 144206 & & $v$ Her & & 12013 & 3.73 & & 10 & NOT & NOT & NOT \\
\hline 145389 & & $\phi$ Her & SB1 & 11781 & 3.97 & & 9.5 & NOT & NOT & NOT \\
\hline 149121 & & $28 \mathrm{Her}$ & & 10908 & 3.83 & & 9 & CAT, NOT & CAT, NOT & \\
\hline \multirow[t]{2}{*}{158704} & A & HR 6520 & SB2 & 13163 & 4.22 & 8.5 & 2.8 & CAT(3) & CAT(2) & CAT \\
\hline & B & & & 8000 & 4.00 & & 14 & & & \\
\hline 165493 & & HR 6759 & SB1 & 13890 & 3.90 & & 2.5 & CAT(3) & CAT & CAT(2) \\
\hline \multirow[t]{2}{*}{174933} & A & 112 Her & SB2 & 13294 & 4.15 & 7.5 & 5 & CAT(2), NOT & CAT, NOT & NOT \\
\hline & B & & & 8500 & 4.20 & & 8 & & & \\
\hline 175640 & & HR 7143 & & 12077 & 3.92 & & 0.5 & CAT(2), NOT(3) & CAT(2), NOT(3) & CAT(2) \\
\hline 178065 & & HR 7245 & SB1 & 12193 & 3.54 & & 0 & CAT(2) & CAT & CAT(2) \\
\hline 182308 & & HR 7361 & & 13570 & 3.56 & & 8 & NOT(2) & NOT(2) & \\
\hline 186122 & & $46 \mathrm{Aql}$ & & 12914 & 3.74 & & 0 & CAT & CAT & CAT \\
\hline \multirow[t]{2}{*}{191110} & A & HR 7694 & SB2 & 12000 & 4.07 & $1.3^{c}$ & 8 & CAT & CAT & \\
\hline & B & & & 11500 & 4.10 & & 8 & & & \\
\hline 193452 & & HR 7775 & SB1 & 10750 & 4.00 & & 1 & CAT, NOT(4) & NOT(4) & CAT, NOT \\
\hline 213236 & & $56 \mathrm{Aqr}$ & & 11977 & 4.06 & & 25 & CAT(2) & & \\
\hline 221507 & & $\beta \mathrm{Scl}$ & & 12476 & 4.13 & & 25 & CAT(2) & & \\
\hline
\end{tabular}

${ }^{a}$ Adopted luminosity ratio at $\lambda 3984$.

${ }^{b}$ The telescope used for the observations of the key wavelength regions. The number within parentheses denotes the number of observations.

${ }^{c}$ Luminosity ratio derived at $\lambda 4046$.

constructed to test the mutual interplay of turbulent and rotational velocities at small values, one utilizing an atmospheric model at the cool end of the $\mathrm{HgMn}$ star $T_{\text {eff }}$ range (represented by HR 7775 with $T_{\text {eff }}=10750 \mathrm{~K}$ ), and one for a hotter model (represented by $\kappa \mathrm{Cnc}$ with $T_{\text {eff }}=13470 \mathrm{~K}$ ). Calculations were made for $\xi_{\mathrm{t}}: v \sin i$ combinations of $0: 2,1: 1$, and 2:0, with all numbers being in units of $\mathrm{km} \mathrm{s}^{-1}$. For small changes in $\xi_{\mathrm{t}}$ and $v \sin i$ their effect is quite similar on the line shape and equivalent width. The calculations were made for two different isotope mixtures, the terrestrial mixture and pure ${ }^{204} \mathrm{Hg}$, typically the extremes considered for the study of mercury isotopes. For a star with a pure ${ }^{204} \mathrm{Hg}$ mixture and a mercury abundance of 
Table 2. Hg lines used in the synthetic spectra.

\begin{tabular}{|c|c|c|}
\hline Isotope & $\lambda(\AA)$ & $\log g f^{a}$ \\
\hline \multicolumn{3}{|c|}{$\mathrm{Hg}$ II $6 \mathrm{~s}^{2}{ }^{2} \mathrm{D}_{5 / 2}-6 \mathrm{~s} 6 \mathrm{p}^{2} \mathrm{P}_{3 / 2}$} \\
\hline${ }^{196} \mathrm{Hg}$ & 3983.7710 & -4.550 \\
\hline${ }^{198} \mathrm{Hg}$ & 3983.8386 & -2.730 \\
\hline${ }^{199 a} \mathrm{Hg}$ & 3983.8437 & -2.880 \\
\hline${ }^{199 b} \mathrm{Hg}$ & 3983.8527 & -2.740 \\
\hline${ }^{200} \mathrm{Hg}$ & 3983.9124 & -2.370 \\
\hline${ }^{201 a} \mathrm{Hg}$ & 3983.9316 & -3.050 \\
\hline${ }^{201 b} \mathrm{Hg}$ & 3983.9414 & -2.810 \\
\hline${ }^{202} \mathrm{Hg}$ & 3983.9932 & -2.250 \\
\hline${ }^{204} \mathrm{Hg}$ & 3984.0724 & -2.880 \\
\hline \multicolumn{3}{|c|}{$\mathrm{Hg}$ I $6 \mathrm{~s} 6 \mathrm{p}^{3} \mathrm{P}_{1}-6 \mathrm{~s} 7 \mathrm{~s}^{3} \mathrm{~S}_{1}$} \\
\hline${ }^{199 a} \mathrm{Hg}$ & 4358.1749 & -2.200 \\
\hline${ }^{201 a} \mathrm{Hg}$ & 4358.2276 & -2.180 \\
\hline${ }^{201 b} \mathrm{Hg}$ & 4358.2410 & -2.210 \\
\hline${ }^{201 c} \mathrm{Hg}$ & 4358.288 & -2.900 \\
\hline${ }^{199 b} \mathrm{Hg}$ & 4358.3145 & -1.500 \\
\hline${ }^{201 d} \mathrm{Hg}$ & 4358.316 & -2.700 \\
\hline${ }^{204} \mathrm{Hg}$ & 4358.320 & -1.620 \\
\hline${ }^{202} \mathrm{Hg}$ & 4358.326 & -0.990 \\
\hline${ }^{200} \mathrm{Hg}$ & 4358.332 & -1.110 \\
\hline${ }^{198} \mathrm{Hg}$ & 4358.337 & -1.470 \\
\hline${ }^{196} \mathrm{Hg}$ & 4358.341 & -3.290 \\
\hline${ }^{201 e} \mathrm{Hg}$ & 4358.3529 & -1.810 \\
\hline${ }^{201 f} \mathrm{Hg}$ & 4358.3619 & -2.210 \\
\hline${ }^{199 c} \mathrm{Hg}$ & 4358.3795 & -1.890 \\
\hline${ }^{201 g} \mathrm{Hg}$ & 4358.4423 & -2.180 \\
\hline${ }^{199 d} \mathrm{Hg}$ & 4358.5196 & -2.200 \\
\hline \multicolumn{3}{|c|}{$\mathrm{Hg}$ II $6 \mathrm{~s} 7 \mathrm{~s}^{2} \mathrm{~S}_{1 / 2}-6 \mathrm{~s} 7 \mathrm{p}^{2} \mathrm{P}_{3 / 2}$} \\
\hline${ }^{199 a} \mathrm{Hg}$ & 6149.419 & -0.881 \\
\hline${ }^{201 a} \mathrm{Hg}$ & 6149.451 & -0.791 \\
\hline${ }^{204} \mathrm{Hg}$ & 6149.461 & -0.837 \\
\hline${ }^{202} \mathrm{Hg}$ & 6149.469 & -0.198 \\
\hline${ }^{200} \mathrm{Hg}$ & 6149.477 & -0.309 \\
\hline${ }^{198} \mathrm{Hg}$ & 6149.483 & -0.672 \\
\hline${ }^{199 b} \mathrm{Hg}$ & 6149.504 & -0.643 \\
\hline${ }^{201 b} \mathrm{Hg}$ & 6149.513 & -0.925 \\
\hline
\end{tabular}

${ }^{a}$ Terrestrial isotopic mixture.

$\log N_{\mathrm{Hg}}=+5.80 \mathrm{dex}$ (on a scale where $\log N_{\mathrm{H}}=12.00$ ), an increase of $1 \mathrm{~km} \mathrm{~s}^{-1}$ in $\xi_{\mathrm{t}}$ prompted a 0.5 dex decrease in the abundance of the $\lambda 3984$ line to obtain the same line depth. In the runs made for a terrestrial mixture the change in abundance was much less, but still noticable (approximately $0.2 \mathrm{dex}$ ). For $\mathrm{Hg}$ I $\lambda 4358$ a $1 \mathrm{~km} \mathrm{~s}^{-1}$ increase in $\xi_{\mathrm{t}}$ lead to a $0.15 \mathrm{dex}$ abundance decrease for the pure ${ }^{204} \mathrm{Hg}$ mixture and 0.05 dex for the terrestrial mixture. The calculations for the $\mathrm{Hg}$ II $\lambda 6149$ line were completely insensitive to a $\xi_{\mathrm{t}}$ increase at this level.

In $\mathrm{HgMn}$ stars the $\mathrm{Hg}$ II $\lambda 3984$ line is often the singular line used for the determination of the mercury abundance. Since this line is affected by small changes in $\xi_{\mathrm{t}}$, the adopted microturbulence is important to the accuracy of the mercury abundance and isotopic mixture derived. Also, since mercury lines of different strengths are affected differently by microturbulence an improper $\xi_{\mathrm{t}}$ could either prevent the detection, or create the appearance, of excitation and ionization anomalies.

Under the assumption that the $g f$ values of the $\mathrm{Hg}$ II $\lambda 3984$ and $\lambda 6149$ lines are correct, the study of these lines can be used as a tool for determining the microturbulent velocity or perhaps be an indicator of the degree to which $\mathrm{HgMn}$ star atmospheres deviate from each other, as for example, in terms of of abundance stratification. As previously noted the $\lambda 6149$ line is virtually unaffected by changes in $\xi_{\mathrm{t}}$, while the stronger $\lambda 3984$ line is affected noticeably. Thus, the $\xi_{\mathrm{t}}$ parameter can be determined by altering its value in the synthetic calculations until the mercury abundance derived from the two lines is the same. Since both lines are from the same ion and originate from high, albeit different, energy levels, the abundance established from the lines should be similar.

The tests show that for the $\mathrm{Hg}$ II $\lambda 3984$ line the isotope mixture, as well as the abundance, is altered as $\xi_{\mathrm{t}}$ is changed. Specifically, the amount of less abundant isotopes increases relative to the more abundant isotopes as $\xi_{\mathrm{t}}$ is increased. In a sharp-lined star a change of $1 \mathrm{~km} \mathrm{~s}^{-1}$ in $\xi_{\mathrm{t}}$ require a corresponding change in the amount of a low abundance isotope by more than $30 \%$ in order to obtain a similar line depth. It is therefore important to compare the values of $\xi_{\mathrm{t}}$ when comparing the mercury abundance and isotopic compositions calculated by different authors. In many of the previous studies of mercury in $\mathrm{HgMn}$ stars $\xi_{\mathrm{t}}$ has been assumed to be zero, which can have introduced systematic errors in the determinations of the mercury abundance and isotopic mixture.

\section{Results}

\subsection{Comments on individual stars}

The $\mathrm{Hg}$ II $\lambda 6149$ line has been investigated in a sample of $\mathrm{HgMn}$ stars for the first time. This line is a useful addition in the study of mercury since its weakness makes it less sensitive than the $\mathrm{Hg}$ II $\lambda 3984$ line to the effects of microturbulence. The proximity of the line to a strong Fe II feature makes the analysis complicated for stars with broader lines ( $\left.v \sin i \geq 10 \mathrm{~km} \mathrm{~s}^{-1}\right)$, as the two features blend together. However, for these stars information about the $\mathrm{Hg}$ II $\lambda 6149$ line can still be ascertained by studying the synthetic fit to the Fe II lines at $\lambda \lambda 6147,6149$.

For at least four of the coolest and most sharp-lined HgMn stars (HR 7775, $\chi$ Lupi, HD 124740 and HR 3302) the $\mathrm{Hg}$ II $\lambda 6149$ line is slightly blueshifted, and there is a suggestion of a similar shift in many of the other HgMn stars. For several stars the reality of the shift cannot be established due to either low mercury abundance or high $v \sin i$. In Fig. 1 a synthetic spectrum is compared to the observed spectrum of $\operatorname{HR} 7775$, illustrating the slight wavelength shift of the Hg II $\lambda 6149$ line. The basis of the wavelength shift is the accurately determined laboratory wavelengths of the nearby Fe II $\lambda \lambda$ 6147.736, 6149.249 features. The observed shifts are not explainable by variations of the isotopic compositions, 
as determined by synthetic spectrum calculations. The presence of a wavelength shift can be a reflection of an error in the applied wavelengths for the isotopes in the $\mathrm{Hg}$ II line or of errors in the Fe II laboratory wavelengths. The latter explanation is considered to be unlikely since the error of the Fe II wavelengths are believed to be better than $2 \mathrm{~m} \AA$ (Johansson 2002, private communication), while the observed shifts are on the

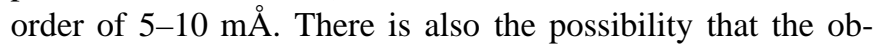
served wavelength shift could be the result of a blending feature contributing to the observed $\mathrm{Hg}$ II line profile. We have not found any mention in the literature of a spectral feature near the $\mathrm{Hg}$ II feature that could be strong enough to alter the observed line profile. The fact that no spectral feature has been observed near the wavelength of the $\mathrm{Hg}$ II $\lambda 6149$ line in several of the Am stars studied in Dolk et al. (2002) is evidence against the blending hypothesis. Also, there is no evidence of a spectral feature in the $\mathrm{HgMn}$ star $46 \mathrm{Aql}$, a star which has a mercury abundance which is too low to produce a $\mathrm{Hg}$ II feature at this wavelength. In our abundance determinations the presence of the wavelength shift was neglected and the mercury abundance was determined by shifting the observed spectrum to fit the synthetic line profile.

The abundance derived from the three optical region mercury lines studied is presented in Table 3 along with measured equivalent widths. In Table 4 the derived mercury abundance and microturbulence is compared to determinations from previous work, and in Table 5 the isotopic mixture derived from the $\mathrm{Hg}$ II $\lambda 3984$ line is presented. The abundances are given as $\log N_{\mathrm{Hg}}$ on a scale where $\log N_{\mathrm{H}}=12$. For all stars the abundance and the isotope mixture were determined iteratively by making a number of synthetic runs for the $\lambda 3984$ line until the best fits were determined. The abundance from the two other mercury lines was determined utilizing the isotopic mixture determined from the $\lambda 3984$ line since the small isotopic separations of these lines do not allow for an accurate determination of the isotopic mixture. According to Michaud et al. (1974) the effect of diffusion would cause a cloud of mercury to build up high up in the HgMn star atmosphere. It is conceivable that lines originating from different excitation levels and ionization stages are formed at various heights in the mercury cloud. This could lead to the isotope of a specific mercury line to be dependant on where it is formed. For this reason it is possible that our use of the $\mathrm{Hg}$ II $\lambda 3984$ isotopic mixture for the $\mathrm{Hg}_{\mathrm{I}} \lambda 4358$ and $\mathrm{Hg}$ II $\lambda 6149$ lines introduces errors in the abundance determinations from these lines. However, the small isotopic separations of $\mathrm{Hg}_{\mathrm{I}} \lambda 4358$ and $\mathrm{Hg}$ II $\lambda 6149$ means that the abundance derived from these lines is quite insensitive to the adopted isotope mixture. Synthetic calculations show that the difference in abundance for the two extremes of the isotope mixture, terrestrial and pure ${ }^{204} \mathrm{Hg}$, is on the order of $0.1 \mathrm{dex}$ for both the $\mathrm{Hg}$ I $\lambda 4358$ and $\mathrm{Hg}$ II $\lambda 6149$ lines.

In $\mathrm{HR} 7775$ there are signs that the $\mathrm{Hg}_{\mathrm{I}} \lambda 4358$ and $\mathrm{Hg}$ II $\lambda 3984$ lines have different isotope mixtures. The isotope mixture measured from the $\lambda 3984$ line does not fit the $\lambda 4358$ feature as well as a mixture consisting of all isotopes, an observation that was previously noted by Wahlgren et al. (2000). The confirmation of a difference in the isotopic structure of mercury lines from different ionization stages would be an important

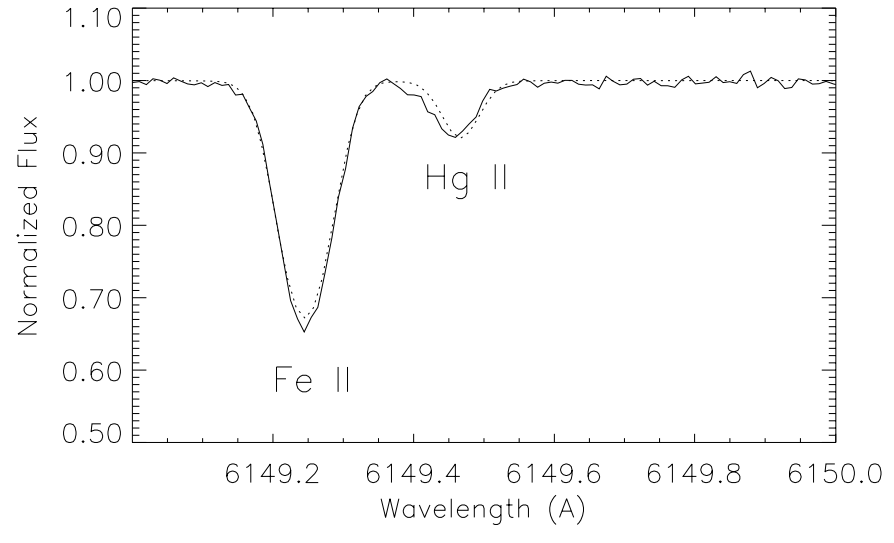

Fig. 1. A synthetic spectrum (dotted) fitted to the observed spectrum of HR 7775 (solid), illustrating the wavelength shift of the $\mathrm{Hg}$ II $\lambda 6149$ line.

constraint on mechanisms proposed to cause the anomalous behaviour of mercury. The difficulty in establishing the reality of this effect from the observed lines means that other lines should be investigated.

In Table 4 the microturbulence established from a comparison between the $\mathrm{Hg}_{\text {II }} \lambda 3984$ and $\lambda 6149$ lines is given. Previously determined values, established from the analysis of $\mathrm{Fe}_{\mathrm{I}}$ and $\mathrm{Fe}$ II lines, were used in our study for stars lacking data for the $\lambda 6149$ region. In our determinations $\xi_{\mathrm{t}}$ was altered until the abundance of the two Hg II lines matched. For HR 7143 and HR 7245, the abundance derived from the $\lambda 3984$ and $\lambda 6149$ lines could not be made to agree for any combination of $\xi_{\mathrm{t}}$ and $v \sin i$. Also, it was noted that the previously determined value of $\xi_{\mathrm{t}}$ for the two stars $\left(1 \mathrm{~km} \mathrm{~s}^{-1}\right)$ was too high, with the synthetic line profiles being broader than the observed spectral features for a $v \sin i$ of $0 \mathrm{~km} \mathrm{~s}^{-1}$. We suggest that the observed discrepancy may be the result of the weakness of the $\lambda 6149$ line in HR 7143 and HR 7245 rather than an increased strength of $\lambda 3984$. This could result from the $\lambda 6149$ feature being partially filled-in because of emission. In the He-weak CP star 3 Cen A the $\lambda 6149$ line has been reported to be in emission (Sigut et al. 2000). Wahlgren \& Hubrig (2000), on the subject of emission lines in late-B type stars, noted that HR 7143 and HR 7245 were the two stars with the strongest $\mathrm{Ti}$ II and $\mathrm{Cr}$ II emission in their sample. It is thus suspected that the highlyexcited $\mathrm{Hg}$ II $\lambda 6149$ line could be affected by emission in these stars.

Of the observed sharp-lined $\mathrm{HgMn}$ stars $(v \sin i \leq$ $10 \mathrm{~km} \mathrm{~s}^{-1}$ ) in this study and that of Woolf \& Lambert (1999a) only two, HR 7361 and $\kappa$ Cnc, have terrestrial-like isotope mixtures, as determined from the $\mathrm{Hg}$ II $\lambda 3984$ line. Our observations of HR 7361 indicate that the isotope mixture is very close to terrestrial. The isotopic mixture noted by Woolf $\&$ Lambert is slightly weighted towards the heavier isotopes, but since HR 7361 has a $v \sin i>5 \mathrm{~km} \mathrm{~s}^{-1}$, the individual isotopes cannot be discerned. The isotope mixture of the star $\kappa \mathrm{Cnc}$ has been noted as terrestrial (Wahlgren et al. 1995) from the analysis of the $\mathrm{Hg}$ II $\lambda 1942$ line, but our analysis of the $\lambda 3984$ line shows a small but noticeable enhancement of the heavier isotopes, a fact which is not substantiated by the mixture given in 
Woolf \& Lambert, which is close to terrestrial. Two of the broader-lined stars of our study, 56 Aqr and $\theta$ Hyi, have isotope mixtures that are close to terrestrial, but their higher $v \sin i$ means that the exact mixture is uncertain. From Table 5 we see that there is no clear trend of the isotope mixture changing with the effective temperature of the star. However, there may be a tendency for the coolest $\mathrm{HgMn}$ stars to have a mixture dominated by the two heaviest mercury isotopes.

In Fig. 2, a selection of $\mathrm{Hg}$ II $\lambda 3984$ line profiles are presented for the sharpest-lined $\left(v \sin i<5 \mathrm{~km} \mathrm{~s}^{-1}\right) \mathrm{HgMn}$ stars of our sample to illustrate the diversity of the observed isotope mixtures. In this figure the stars are arranged by the relative contribution of the ${ }^{204} \mathrm{Hg}$ feature. Also included in the figure is the best-fit synthetic spectrum for each star, as well as the position of the isotopic and hyperfine components.

The star HR 6759 has a rather peculiar isotope mixture, where the ${ }^{198} \mathrm{Hg}$ and ${ }^{199} \mathrm{Hg}$ isotopes are present along with ${ }^{202} \mathrm{Hg}$ and ${ }^{204} \mathrm{Hg}$, while the ${ }^{200} \mathrm{Hg}$ and ${ }^{201} \mathrm{Hg}$ isotopes are almost completely lacking (Fig. 2). This mixture is somewhat similar to that observed for 11 Per (Woolf \& Lambert 1999a) and is difficult to explain using radiative diffusion, since isotopic mass differences alone cannot account for these observed mixtures. Both stars are among the hottest HgMn stars with effective temperatures of $13890 \mathrm{~K}$ (HR 6759) and $14550 \mathrm{~K}$ (11 Per). The existence of another spectral feature at the position of the ${ }^{199} \mathrm{Hg}$ isotope would be a possible explanation, but there currently does not exist independent evidence from non- $\mathrm{HgMn}$ stars to support this claim. The presence of a binary companion could conceivably also render the observed line profiles without a peculiar isotope mixture, but the fact that the line profile is the same in two of the observations taken years apart suggests that this is not the case.

In the spectrum of HR 7694 lines from both components of the binary were observed in the region 4017-4053 $\AA$. The strength of these lines indicate that the primary to secondary star luminosity ratio is approximately $L_{A} / L_{B}=1.3$. At the time of this observation the wavelength separation was $\lambda_{A}-\lambda_{B}=$ $-0.90 \pm 0.1 \AA$, corresponding to a velocity shift of $67 \mathrm{~km} \mathrm{~s}^{-1}$. An observation of the region 3964-3998 $\AA$ was obtained on the following evening under the unfortunate circumstance that the relative radial velocity between the two components was essentially zero. Because of this, the lines from the two components coincide in wavelength. According to Dworetsky (1974) the primary star has strong $\mathrm{Hg}$ II $\mathbf{1 3 9 8 4}$, and we interpret his statements to imply that the secondary also presents this line. The wavelength coincidence of the two spectra makes it impossible to get an accurate estimate of the individual mercury abundance and isotopic mixture in the two stars. However, synthetic fitting of the combined spectral feature indicates that the $\mathrm{Hg}$ II $\lambda 3984$ feature is strong in both stars and that the isotopic mixtures are weighted towards the heaviest isotopes. A reasonably good fit to the feature was obtained with the aid of the isotopic-fractionation model of White et al. (1976), utilizing the mix parameters $q=1$ and $q=0.5$ for the primary and secondary, respectively. The abundances obtained utilizing this model are listed in Table 3.

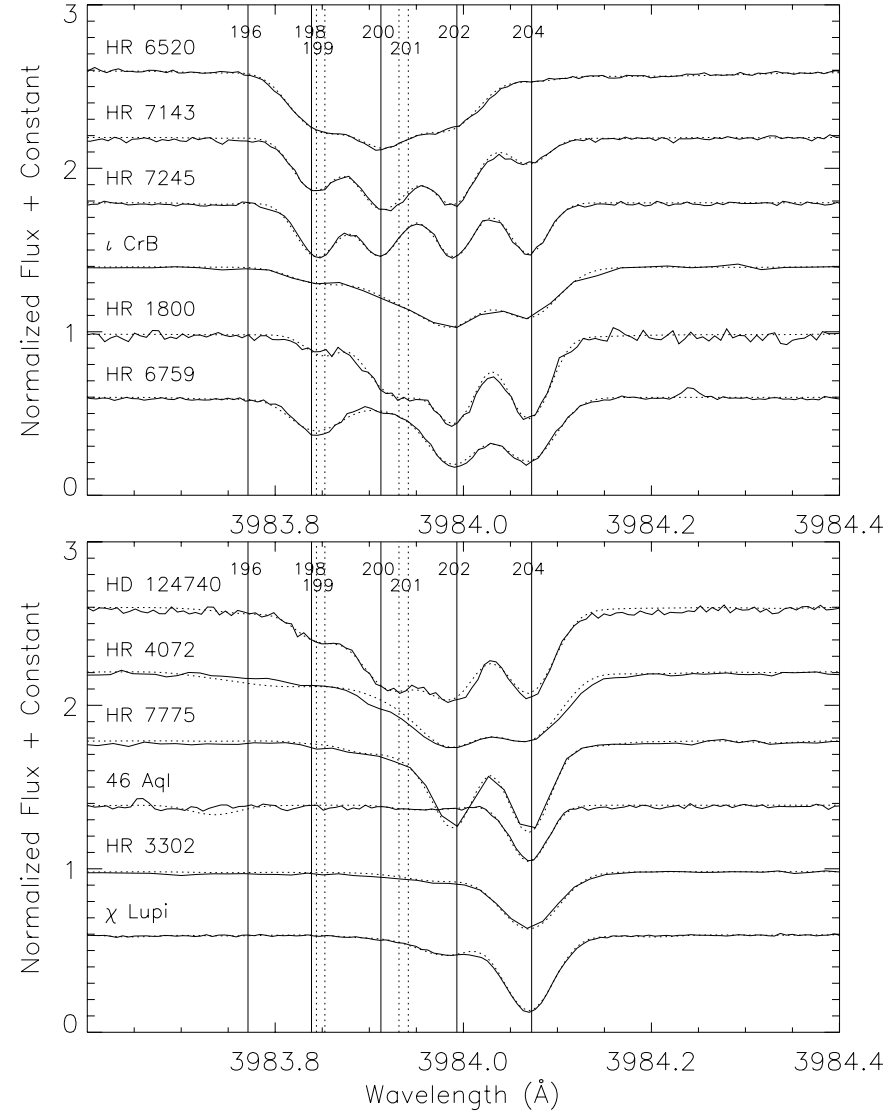

Fig. 2. Observed spectra of sharp-lined HgMn stars plotted with the best-fit synthetic isotope mixture. The stars are ordered by the relative strength of the ${ }^{204} \mathrm{Hg}$ feature. The solid and dotted vertical lines indicate the wavelengths for the isotope and hyperfine components, respectively.

\subsection{Search for abundance trends}

From the studied stars there are several abundance trends that can be noted. One such trend is that the mercury abundance derived from the $\mathrm{Hg}$ I $\lambda 4358$ line is lower than the abundance derived from the $\mathrm{Hg}_{\mathrm{II}}$ lines by an amount approximately equivalent to the sum of the respective errors in the seven stars in which the $\lambda 4358$ line is observed with certainty. The apparent discrepancy in the abundance derived from the mercury lines could be indicative of an ionization anomaly, but could also indicate problems with the atomic data. The observation of other $\mathrm{Hg}$ I and $\mathrm{Hg}$ II lines could establish the reality of the ionization anomaly. Accurate laboratory analysis for the oscillator strengths of these lines is also encouraged.

Questions have been raised by Hubrig \& Mathys (1995) as to whether specific elements could be concentrated differently on the stellar surface of HgMn stars. They remarked that the mercury abundance is large and manganese abundance low in several $\mathrm{HgMn}$ spectroscopic binaries whose orbital plane has a small inclination to the line of sight, while the situation is reversed in binaries with the orbital plane perpendicular to the line of sight. Since close binaries typically have their spin axes aligned perpendicular to the orbital plane, this would suggest that manganese would be concentrated towards the rotational poles, while mercury would gravitate towards the equatorial 


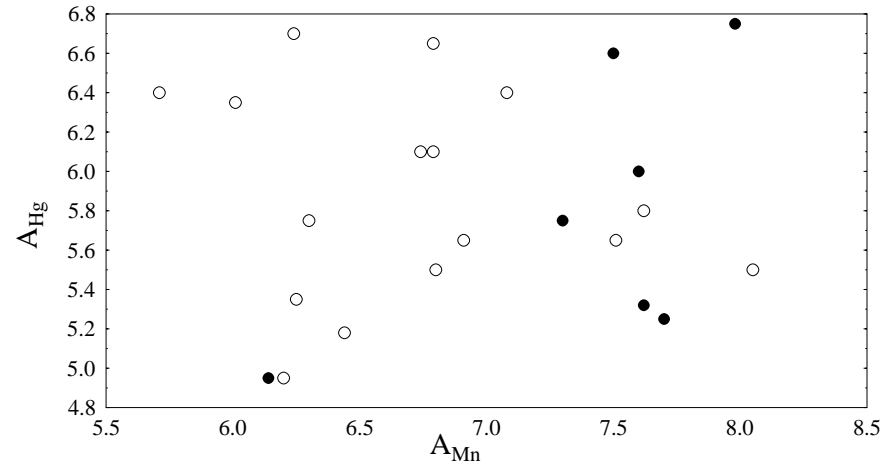

Fig. 3. The abundance of mercury plotted against the abundance of maganese, with open circles representing sharp-lined stars and filled circles broader-lined stars.

region. New observations utilizing Doppler imaging have shown that mercury is primarily concentrated towards the equatorial region in the $\mathrm{HgMn}$ star $\alpha$ And (Wahlgren et al. 2001; Adelman et al. 2002), lending credit to the claim of inhomogeneous surface distributions for, at least, one element.

In Fig. 3 mercury abundances derived from $\mathrm{Hg}_{\text {II }} \lambda 3984$ (from the present work and by Woolf \& Lambert 1999a) have been plotted against the manganese abundances to investigate a possible difference in surface distribution of these elements. The manganese abundance is for most stars taken from Jomaron et al. (1999), and for two stars (41 Eri A and B) it was derived during the current investigation. From Fig. 3 it is evident that there is no striking anticorrelation between mercury and manganese It is worth noting that while the manganese abundance increases noticeably with the $T_{\text {eff }}$ of the star (Smith \& Dworetsky 1993; Jomaron et al. 1999), the mercury abundance does not display a similar $T_{\text {eff }}$ dependence. It can be questioned whether stars of different $T_{\text {eff }}$ should be used in a comparison like Fig. 3 since the abundance- $T_{\text {eff }}$ dependence can be quite different for the elements that are compared. Thus, the neglect of stellar $T_{\text {eff }}$ in an abundance comparison could create or remove the appearance of an abundance correlation. Therefore, it might be preferable to compare the abundances in $\mathrm{HgMn}$ stars of similar $T_{\text {eff }}$ in order to validate abundance correlations. The limited number of stars that share a simi$\operatorname{lar} T_{\text {eff }}$ in our sample precludes us from pursuing this kind of an investigation.

If an anticorrelation between mercury and manganese can be validated, it might be expected that other abundance correlations or anticorrelations exist in $\mathrm{HgMn}$ stars. Hubrig et al. (2003) present early evidence of an iron - manganese anticorrelation in HgMn stars, implying that these elements predominantely exist at different places in the HgMn star atmosphere. The recent results notwithstanding, evidence of inhomogeneous surface distributions of elements in $\mathrm{HgMn}$ stars is currently sparse and further studies should be undertaken to investigate this phenomenon.

If abundance inhomogenieties are distributed as patches on the stellar surface, one might expect to observe differences in the equivalent width of the spectral features. For several stars multiple observations were made of the $\mathrm{Hg}$ II $\lambda 3984$ feature. The result of the measurements for these stars did not find any change in the equivalent width that were larger than the approximated measurement error (as defined by the noise level and the fitting procedure to be $2-3 \mathrm{~m} \AA$ ). The measurements do not, however, completely rule out the possibilty of mercury patches on the HgMn stars, since the existence of patches would not necessarily have an effect on the equivalent width. Our results notwithstanding additional investigations into the existence of line profile variability in $\mathrm{HgMn}$ star spectra should be undertaken.

While studies of $\mathrm{HgMn}$ stars have shown a correlation between $T_{\text {eff }}$ and manganese abundance (Smith \& Dworetsky 1993; Jomaron et al. 1999), no clear correlation has been found between $T_{\text {eff }}$ and the mercury abundance (Smith 1997; Woolf \& Lambert 1999a). Woolf \& Lambert noted a possible decrease in $\mathrm{A}(\mathrm{Hg})$ with $T_{\text {eff }}$ for sharp-lined HgMn stars. In Figs. 4a, b the mercury abundance from the present work and from Woolf \& Lambert, is plotted against $T_{\text {eff }}$, with Fig. 4a showing the sharp-lined stars $\left(v \sin i \leq 10 \mathrm{~km} \mathrm{~s}^{-1}\right)$, and Fig. 4b showing the entire sample of $\mathrm{HgMn}$ stars from the two studies. From Fig. $4 \mathrm{a}$ a weak trend of the mercury abundance decreasing with increasing $T_{\text {eff }}$ is evident, while no trend can be detected in Fig. $4 \mathrm{~b}$ when the broader-lines stars are included. The fact that Fig. $4 \mathrm{~b}$ fails to show any trend could be attributed to the substantially larger errors associated with the determined mercury abundance of the broader-lined stars. In the broader-lined stars the determination of the isotopic composition is very uncertain, which consequently causes larger uncertainties in the determined mercury abundance.

\subsection{Error analysis}

There are several sources of errors for the mercury abundances given in Table 3, only some of which are included in the stated uncertainties. The uncertainties presented in Table 3 are rms errors which were calculated for each star and each mercury line by taking into account the effect of uncertainties in the stellar parameters, isotope mixtures, and synthetic spectrum fitting. The uncertainties of the stellar parameters were taken to be $\Delta T_{\text {eff }}= \pm 200 \mathrm{~K}, \Delta \log g= \pm 0.1 \mathrm{dex}$, and $\Delta \xi_{\mathrm{t}}= \pm 0.5 \mathrm{~km} \mathrm{~s}^{-1}$. The uncertainty in $T_{\text {eff }}$ gives an error of approximately \pm 0.03 dex for the $\mathrm{Hg}$ II $\lambda 3984$ and $\lambda 6149$ lines, and of \pm 0.05 dex for the $\mathrm{Hg}_{\mathrm{I}} \lambda 4358$ line. The uncertainty in $\log g$ results in an error of \pm 0.01 dex for the two $\mathrm{Hg}$ II lines and of \pm 0.02 dex for the $\mathrm{Hg}$ I line. A change in the microturbulent velocity affects the three mercury lines very differently (as noted in Sect. 3.3). For $\mathrm{Hg}$ II $\lambda 3984$ a $0.5 \mathrm{~km} \mathrm{~s}^{-1}$ change in $\xi_{\text {t }}$ gives rise to an abundance change of 0.1 to 0.25 dex depending on the isotope mixture, while a similar change gives rise to a $0.05 \mathrm{dex}$ change in the $\mathrm{Hg}$ I $\lambda 4358$ abundance and no discernable change in the $\mathrm{Hg}$ II $\lambda 6149$ abundance. In the rms uncertainty of the $\mathrm{Hg}$ II $\lambda 3984$ abundance the error arising from $\xi_{\mathrm{t}}$ has been estimated to be $0.1,0.175$ and 0.25 dex, depending on whether the mercury isotope mixture is terrestrial-like, more weighted towards the heavier isotopes, or essentially pure ${ }^{204} \mathrm{Hg}$, respectively. For the $\mathrm{Hg}$ I $\lambda 4358$ line a 0.05 dex abundance error was utilized to account for the $\xi_{\mathrm{t}}$ uncertainty in all stars. 

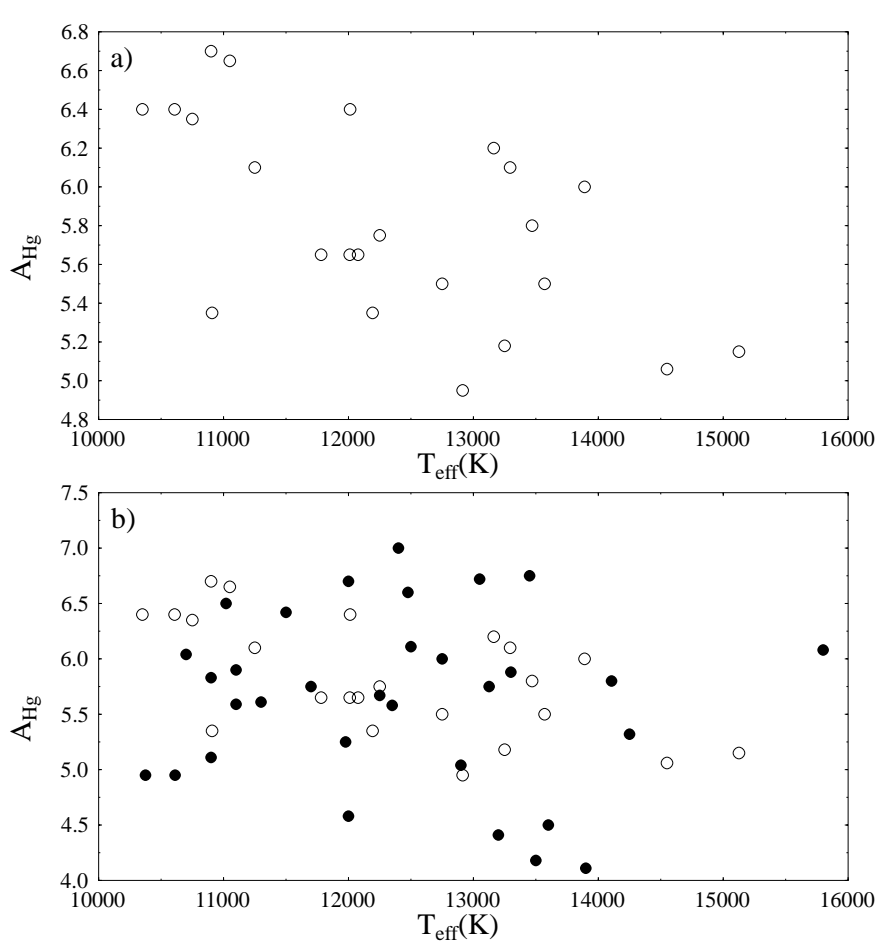

Fig. 4. The abundance of mercury plotted against $T_{\text {eff }}$, with a) showing the sharp-lined stars $\left(v \sin i \leq 10 \mathrm{~km} \mathrm{~s}^{-1}\right)$ and $\left.\mathbf{b}\right)$ the entire sample of $\mathrm{HgMn}$ stars with open circles representing the sharp-lined stars and filled circles the broader-lined stars.

The accuracy of the derived mercury abundance is dependent on errors in the adopted isotope mixture. This is particularly noticeable for the $\mathrm{Hg}$ II $\lambda 3984$ line where the individual isotopes are clearly separated in wavelength. For stars with $v \sin i \leq 5 \mathrm{~km} \mathrm{~s}^{-1}$ the lines from individual isotopes can be discerned, and the isotopic mixture uncertainty is subsequently quite small. For stars with $v \sin i \geq 5 \mathrm{~km} \mathrm{~s}^{-1}$, however, the individual isotopes cannot be separated and the uncertaintity in the isotopic mixture, and therefore the derived mercury abundance, is larger. The effect of the isotopic mixture uncertainties on the derived mercury abundance was investigated by calculating synthetic spectra for different mixtures that still gave reasonable fits to the observed spectra. The results of these rather subjective tests showed that the uncertainties of the isotope mixtures produced a difference in the mercury abundance between 0.05 to $0.4 \mathrm{dex}$, with the error becoming progressively larger with increasing $v \sin i$. The isotope mixture uncertainties have been estimated for each star individually and included in the stated uncertainties of the $\mathrm{Hg}_{\text {II }} \lambda 3984$ abundance. For the Hg I $\lambda 4358$ and $\mathrm{Hg}$ II $\lambda 6149$ lines, which are much less sensitive to the adopted isotope mixtures, an uncertainty of 0.05 and 0.02 dex, respectively, has been utilized to account for the uncertainty in the isotope mixtures.

The spectrum fitting errors include uncertainties arising from noise, continuum placement, and blending of spectral features. These uncertainties were estimated for each star individually by allowing the continuum placement to change by \pm 0.01 and the abundance of blending features to change by \pm 0.2 dex. For $\mathrm{Hg}$ II $\lambda 6149$, the mercury feature is blended with the strong
Fe II $\lambda 6149$ line in stars with $v \sin i \geq 10 \mathrm{~km} \mathrm{~s}^{-1}$, which increases the uncertainty of the derived mercury abundance.

The uncertainty in the isotopic mixture is difficult to estimate since it depends in a complicated way on the errors in parameters such as $v \sin i$ and $\xi_{\mathrm{t}}$, as well as on the mixture itself. To estimate the isotopic mixture uncertainty several synthetic runs were made for each star, varying the continuum and isotopic mixture within values reasonable for each star. The uncertainties in the isotopic mixtures are presented in Table 5. From this table it is observed that the estimated uncertainties are quite small in HgMn stars with $v \sin i \leq 5 \mathrm{~km} \mathrm{~s}^{-1}$. This is due to the fact that most of the isotopes are clearly separated. For the broader-lined stars the uncertainties get progressively larger with increasing $v \sin i$, and for the stars of our sample that have the largest $v \sin i$, the noted isotope mixture can only be used as an approximate measure of the mixture.

Several possible sources of uncertainties are not included in the rms uncertainties. Among these are the possible errors in the stellar parameters and model atmospheres that could arise from the unusual chemical composition of the HgMn stars, the effects of diffusion processes, appropriateness of the LTE assumption, and the presence of patches or magnetic fields. Another possible error source could arise from the neglect of binary components, in particular among the noted SB1 binaries. The magnitude of this error will mostly depend on the luminosity ratio of the components. For example, by ignoring a secondary with a luminosity ten times lower than the primary, one would introduce an error of approximately $0.1-0.2 \mathrm{dex}$ in the mercury abundance determinations.

\subsection{Comparison with previous studies}

One of the motivations for the current investigation was to add to the growing list of $\mathrm{HgMn}$ stars studied for mercury isotopic and/or abundance anomalies. The interest would mainly lie in creating a sufficiently large sample so that trends in the behaviour of mercury can be better studied. It is also informative to compare the results from multiple investigations to check for similarities as well as inconsistencies. In Table 4 a comparison is made of the mercury abundance derived from this study and those of Smith (1997), Woolf \& Lambert (1999a) and Hubrig et al. (1999b). These studies utilized high spectral resolving powers to investigate the isotopic compositions as well as the mercury abundance, making a comparison with the present work possible.

When comparing the present study with that of Woolf \& Lambert it should be remembered that a 0.2 dex larger total $\log g f$ value was used in their work for the $\mathrm{Hg}$ II $\lambda 3984$ line. Also, the adopted wavelengths of some of the isotopic components in this line are slightly different, but these wavelength differences do not produce noticeable differences in the determined isotope mixtures of a specific star. The mercury abundance of Woolf \& Lambert in Table 4 has been reduced by 0.2 dex to account for the difference in the $\log g f$ value between the two studies. For the mercury abundance of most stars the agreement is within 0.3 dex between the two studies, with some notable discrepancies. Among the sharper-lined 
stars $\left(v \sin i \leq 10 \mathrm{~km} \mathrm{~s}^{-1}\right), \phi$ Her and $v$ Her display noticeably different abundances in the two studies, with the value for $\phi$ Her being 0.36 dex larger and $v$ Her 0.38 dex smaller in our study. A comparison of the stellar parameters and isotope mixture used for $\phi$ Her renders no clue to the reason of the discrepancy. For $v$ Her the adopted microturbulence in the two studies differs by $1 \mathrm{~km} \mathrm{~s}^{-1}$, and the adopted isotopic mixture is noticeably different. These two differences could easily account for the abundance difference. The adopted value in our study of $\xi_{\mathrm{t}}=0 \mathrm{~km} \mathrm{~s}^{-1}$ is based on the comparison of the $\mathrm{Hg}$ II $\lambda 3984$ and $\lambda 6149$ lines yielding the same abundance. Our calculations show that the $\xi_{\mathrm{t}}$ adopted by Woolf $\&$ Lambert would produce a difference of 0.4 dex in the abundance derived from the two lines. For the star $\kappa$ Cnc the 1 dex discrepancy of the mercury abundance is even more pronounced and cannot be explained by the small differences in stellar parameters and the isotopic mixture. The abundance from $\mathrm{Hg}$ II $\lambda 3984$ in this paper is supported by the $\lambda 6149$ abundance as well as the abundance derived in other studies (Smith 1997; Wahlgren et al. 1995). The discrepancy in the mercury abundance of $v$ Cnc between the two studies is also very high ( $0.73 \mathrm{dex})$, but cannot be fully investigated since the isotope mixture is not discussed by Woolf \& Lambert.

Comparisons of the mercury abundance with the study of Smith (1997) also show generally good agreement. There are, however, several stars where the mercury abundance is noticeably different, partially accountable by differences in the adopted stellar parameters, and an additional influence stemming from the isotopic-fractionation model adopted for the isotopic mixture determination by Smith. This model introduces a dimensionless mix parameter, q, to define the isotope mixture (White et al. 1976). Recently, this approach has been found to give inadequate fits to the $\mathrm{Hg}$ II $\lambda 3984$ lines, particularly when very high resolution spectra are used (Proffitt et al. 1999; Woolf \& Lambert 1999a; Hubrig et al. 1999b; Wahlgren et al. 2000). Instead, it has been advocated that a trial and error approach be used, where the isotopic composition is modified until a satisfactory fit is achieved. Despite the difference in approach the basic agreement of most abundance values is an indication that the two studies can be combined to investigate trends in the mercury abundance.

A comparison between our work and that of Woolf \& Lambert shows the derived isotope fractions to be similar for the sharpest-lined stars $\left(v \sin i \leq 5 \mathrm{~km} \mathrm{~s}^{-1}\right)$ that are common to both studies. When they exist, the isotope fraction discrepancies can be accounted for by differences in the adopted stellar parameters, noise effects, or differences in the synthetic fitting routine. It is also possible that the error bars on the isotope fractions given in the two studies are underestimated. In both studies the isotope fraction error bars were determined by repetitive synthetic fitting in which the isotope mixtures and continuum placement was allowed to change. In neither of the studies have uncertainties arising from errors in the stellar parameters been considered, primarily since their effect on the isotopic mixture is very difficult to assess. There is also the interesting possibilty that the noted difference in the isotope fractions reflects a change in the $\mathrm{Hg}$ II $\lambda 3984$ line profile during the time between observations.

\section{Discussion}

The present investigation surveys a sample of HgMn stars for trends and observational properties that could improve our understanding of these objects and aid the theoretical depiction of the $\mathrm{HgMn}$ phenomenon. A number of observations have been made with interesting implications, but the behaviour of mercury in $\mathrm{HgMn}$ stars is far from completely understood. In this section some of the implications from our results are discussed.

The adopted value of the microturbulence parameter can have a large impact on the determined abundance values, especially when strong spectral lines are utilized. The determination of $\xi_{\mathrm{t}}$ is typically accomplished by forcing all the spectral lines from an abundant elemental ion (typically Fe II) to yield the same abundance. The typical assumptions of this approach are that the different lines from a specific ion yield the same abundance, and that the determined $\xi_{\mathrm{t}}$ value is the same for all elements. These assumptions can be questioned as lines from different elements/ions/energy levels can be formed at different heights within the stellar atmosphere and be subjected to different atmospheric conditions and physical effects. Since the $\xi_{\mathrm{t}}$ parameter is typically thought to represent some kind of velocity fields within the atmosphere, it is possible that the $\xi_{\mathrm{t}}$ value would change with atmospheric height, and subsequently be different for different elements and ions. It is also possible that non-LTE effects, errors in atomic data and line blending, introduce significant errors in the determined $\xi_{\mathrm{t}}$ value. In this study the $\mathrm{Hg}$ II $\lambda 6149$ and $\lambda 3984$ lines have been used to provide a measure of the microturbulent velocity. A determination of the microturbulence parameter hinging on only two mercury lines is precarious since an error in the atomic data of one of the lines would yield errors in all the determined $\xi_{\mathrm{t}}$ values. The fact that the mercury abundance determined from the two lines is in agreement for several of the stars for which a null value for the microturbulent velocity has been previously noted is perhaps an indication that the atomic data are of comparable error.

As mentioned in Sect. 4.1, the abundance derived from $\mathrm{Hg}$ II $\lambda 6149$ and $\lambda 3984$ lines cannot be made to agree for any value of $\xi_{\mathrm{t}}$ for the two HgMn stars HR 7143 and HR 7245. Our suggestion is that this can be due to the $\lambda 6149$ feature being partially filled-in because of emission. This raises the question if such a fill-in effect is present in other $\mathrm{HgMn}$ stars of our sample. A partial fill-in of the $\mathrm{Hg}$ II $\lambda 6149$ feature would affect the accuracy of our $\xi_{\mathrm{t}}$ and abundance determination. The fact that HR 7143 and HR 7245 are the two stars, in a sample of nine HgMn stars, which have the strongest $\mathrm{Ti}$ II and $\mathrm{Cr}$ II emission features (Wahlgren \& Hubrig 2000) could be an indication that the fill-in effect of the $\mathrm{Hg}$ II $\lambda 6149$ feature is not significant in most HgMn stars.

Our analysis indicates that there is a systematic difference in the mercury abundance derived from the $\mathrm{Hg}_{\mathrm{I}} \lambda 4358$ and $\mathrm{Hg}$ II $\lambda 3984$ lines for all stars where both lines are strong enough to be observed with certainty. It is by no means certain that this discrepancy can be attributed to an ionization anomaly since uncertainties in the atomic data for one or both of these lines could account for the observed discrepancy. If the larger $g f$ value of Proffitt et al. (1999) is used, the discrepancy would disappear for several of the stars. However, since all of 
Table 3. The abundance of mercury and manganese in $\mathrm{HgMn}$ stars.

\begin{tabular}{|c|c|c|c|c|c|c|c|c|}
\hline \multirow{2}{*}{$\begin{array}{r}\text { Star } \\
\text { name }\end{array}$} & \multirow{2}{*}{$\begin{array}{l}T_{\text {eff }} \\
(\mathrm{K})\end{array}$} & \multicolumn{3}{|c|}{$\log N_{\mathrm{Hg}}$} & \multicolumn{3}{|c|}{$W_{\lambda}(\mathrm{m} \AA)^{a}$} & \multirow[t]{2}{*}{$\log N_{\mathrm{Mn}}^{b}$} \\
\hline & & $\lambda 3984$ & $\lambda 4358$ & $\lambda 6149$ & $\lambda 3984$ & $\lambda 4358$ & $\lambda 6149$ & \\
\hline HD 124740 & 10350 & $6.40 \pm 0.21$ & $6.10 \pm 0.18$ & $6.60 \pm 0.15$ & 118 & 12 & 11 & \\
\hline$v \mathrm{Cnc}$ & 10375 & $4.95 \pm 0.39$ & $<5.15$ & & 39 & $\mathrm{w}$ & bl & \\
\hline$\chi$ Lup & 10608 & $6.40 \pm 0.26$ & $5.90 \pm 0.15$ & $6.40 \pm 0.11$ & 41 & 8.0 & 4.5 & 5.71 \\
\hline$\phi$ Phe & 10612 & $4.95 \pm 0.38$ & $<5.00$ & & 19 & w & & 6.14 \\
\hline HR 7775 & 10750 & $6.35 \pm 0.18$ & $6.05 \pm 0.12$ & $6.50 \pm 0.11$ & 71 & 14 & 5.5 & 6.01 \\
\hline HR 4072 & 10900 & $6.70 \pm 0.21$ & $6.35 \pm 0.14$ & $6.65 \pm 0.13$ & 92 & 13 & 6 & 6.24 \\
\hline $28 \mathrm{Her}$ & 10908 & $5.35 \pm 0.29$ & $<5.40$ & & 29 & w & & 6.25 \\
\hline HR 4487 & 11020 & $6.50 \pm 0.37$ & & & 96 & & & \\
\hline HR 1800 & 11088 & $6.65 \pm 0.18$ & & $6.65 \pm 0.13$ & 92 & & 8.2 & 6.79 \\
\hline$\iota \mathrm{CrB}$ & 11250 & $6.10 \pm 0.18$ & $5.90 \pm 0.15$ & $6.10 \pm 0.14$ & 72 & 7.0 & 4.5 & 6.79 \\
\hline HR 7694 B & 11500 & $6.20 \pm 0.5$ & & & $49^{c}$ & $\mathrm{w}$ & & \\
\hline$\phi$ Her & 11781 & $5.65 \pm 0.23$ & $<5.60$ & $5.70 \pm 0.23$ & 61 & $\mathrm{w}$ & bl & 6.91 \\
\hline $56 \mathrm{Aqr}$ & 11977 & $5.25 \pm 0.37$ & & & 47 & & & 7.7 \\
\hline HR 7694 A & 12000 & $6.70 \pm 0.5$ & & & $73^{c}$ & $\mathrm{w}$ & & \\
\hline HR 3302 & 12010 & $5.65 \pm 0.26$ & & $6.00 \pm 0.17$ & 33 & & 3.3 & \\
\hline$v$ Her & 12013 & $6.40 \pm 0.27$ & & $6.40 \pm 0.23$ & 110 & & bl & 7.08 \\
\hline HR 7143 & 12077 & $5.65 \pm 0.15$ & $<5.50$ & $5.35 \pm 0.16$ & 80 & w & 2.5 & 7.51 \\
\hline HR 7245 & 12193 & $5.35 \pm 0.12$ & & $5.00 \pm 0.18$ & 68 & $\mathrm{w}$ & 2 & \\
\hline 41 Eri B & 12250 & $5.75 \pm 0.29$ & & & 15 & $\mathrm{w}$ & & $6.3^{d}$ \\
\hline AV Scl & 12400 & $7.00 \pm 0.35$ & $6.60 \pm 0.20$ & $7.00 \pm 0.25$ & 117 & 12 & & \\
\hline$\beta \mathrm{Scl}$ & 12476 & $6.60 \pm 0.43$ & & & 127 & & & 7.5 \\
\hline 41 Eri A & 12750 & $5.50 \pm 0.35$ & & & 33 & w & & $6.8^{d}$ \\
\hline$\mu$ Lep & 12750 & $6.00 \pm 0.39$ & $<6.10$ & $6.00 \pm 0.27$ & 115 & w & bl & 7.6 \\
\hline $46 \mathrm{Aql}$ & 12914 & $4.95 \pm 0.26$ & & $<4.90$ & 17 & $\mathrm{w}$ & w & 6.2 \\
\hline 87 Psc & 13126 & $5.80 \pm 0.37$ & & & 108 & & & 7.3 \\
\hline HR 6520 & 13163 & $6.20 \pm 0.14$ & $5.90 \pm 0.20$ & $6.20 \pm 0.12$ & 92 & 3.5 & 8.0 & \\
\hline 112 Her & 13294 & $6.10 \pm 0.20$ & $<6.20$ & $6.10 \pm 0.15$ & 86 & w & bl & 6.74 \\
\hline$\kappa \mathrm{Cnc}$ & 13470 & $5.80 \pm 0.19$ & & $5.85 \pm 0.23$ & 89 & $\mathrm{w}$ & bl & 7.62 \\
\hline HR 7361 & 13570 & $5.50 \pm 0.19$ & & & 85 & $\mathrm{w}$ & & 8.05 \\
\hline HR 6759 & 13890 & $6.00 \pm 0.20$ & $<6.00$ & $5.95 \pm 0.12$ & 74 & $\mathrm{w}$ & 10 & \\
\hline$\theta$ Hyi & 14106 & $5.80 \pm 0.41$ & & & 96 & & & \\
\hline HR 4089 & 15126 & $5.15 \pm 0.23$ & & & 26 & & & \\
\hline
\end{tabular}

${ }^{a}$ The designations "w" and "bl" denote that the spectral line was too weak or blended to measure an equivalent width.

${ }^{b}$ Manganese abundance determined from Jomaron et al. (1999).

${ }^{c}$ Equivalent width determined from synthetic line profile of best fit.

${ }^{d}$ Manganese abundance derived during the current investigation.

the stars do not have equally large discrepancies between the $\mathrm{Hg}$ I and $\mathrm{Hg}$ II abundance, this indicates that the anomaly is not solely the result of a $g f$-value problem. A comparison between the $\mathrm{Hg}_{\mathrm{I}} \lambda 4358$ and $\mathrm{Hg}$ II $\lambda 3984$ abundances in more $\mathrm{HgMn}$ stars, coupled with the investigation of other $\mathrm{Hg}_{\mathrm{I}}$ and $\mathrm{Hg}$ II lines, could decide whether there is an ionization anomaly of mercury in $\mathrm{HgMn}$ stars.

Many of the results of this paper would benefit from increasing the number of observed mercury lines. Such an increase could effectively resolve the reality of the suggeted ionization anomaly, increase the accuracy of the $\xi_{\mathrm{t}}$ and abundance determinations, and aid in resolving whether the weak mercury abundance trends suggested in this paper are real. In the optical and near-infrared region the $\mathrm{Hg}_{\mathrm{I}} \lambda \lambda 5460.7,5769.6$, 5790.7, and the $\mathrm{Hg}$ II $\lambda \lambda 5677.17944 .5$ lines could be investigated. These lines were observed in the cool HgMn star HR 7775 (Wahlgren et al. 2000). In the ultraviolet region there are a large number of $\mathrm{Hg}_{\mathrm{I}}, \mathrm{Hg}_{\text {II }}$ and $\mathrm{Hg}_{\text {III }}$ lines, including the resonance lines of these ionization stages, that could be investigated. There are, however, difficulties in obtaining data in the ultraviolet region at high enough resolving power to produce accurate abundance determinations. An additional problem would be the severe line-blending problems that complicate the analyses of ultraviolet spectra from $\mathrm{HgMn}$ stars.

The mechanisms responsible for producing the chemical peculiarities in $\mathrm{HgMn}$ stars are still unclear. Currently, 
Table 4. Comparison with previous work.

\begin{tabular}{|c|c|c|c|c|c|c|c|c|c|}
\hline \multirow[t]{2}{*}{ Star } & \multirow{2}{*}{$\begin{array}{l}T_{\text {eff }} \\
(\mathrm{K})\end{array}$} & \multicolumn{4}{|c|}{$\mathrm{Hg}$ abundance ${ }^{a}$} & \multicolumn{2}{|c|}{$\xi_{\mathrm{t}}\left(\mathrm{km} \mathrm{s}^{-1}\right)$} & \multirow[t]{2}{*}{ Ref. } & \multirow{2}{*}{$\begin{array}{c}v \sin i \\
\left(\mathrm{~km} \mathrm{~s}^{-1}\right)\end{array}$} \\
\hline & & present & {$[\mathrm{A}]^{b}$} & {$[\mathrm{~B}]$} & {$[\mathrm{C}]$} & present $^{c}$ & prev. & & \\
\hline HD 124740 & 10350 & 6.40 & & & & 0.0 & & & 2 \\
\hline$v \mathrm{Cnc}$ & 10375 & 4.95 & 5.68 & 5.12 & & & 0.1 & {$[1]$} & 19 \\
\hline$\chi$ Lupi & 10608 & 6.40 & & 6.40 & 6.50 & 0.2 & 0.0 & {$[2]$} & 2.1 \\
\hline$\phi$ Phe & 10612 & 4.95 & & 5.12 & & & 0.5 & {$[1]$} & 14 \\
\hline HR 7775 & 10750 & 6.35 & 6.63 & 6.33 & 6.65 & 0.0 & 0.0 & {$[3]$} & 1 \\
\hline HR 4072 & 10900 & 6.70 & 6.84 & 6.72 & & 0.5 & 0.2 & [4] & 3.5 \\
\hline $28 \mathrm{Her}$ & 10908 & 5.35 & 5.51 & 5.69 & & & 0.5 & [3] & 9 \\
\hline HR 4487 & 11020 & 6.50 & & & & $0.0^{d}$ & & & 18 \\
\hline HR 1800 & 11088 & 6.65 & 6.56 & 6.15 & 6.30 & 0.0 & 0.5 & {$[4]$} & 2 \\
\hline$\iota \mathrm{CrB}$ & 11250 & 6.10 & 5.94 & 6.12 & & 0.2 & 0.9 & [3] & 2 \\
\hline HR 7694 b & 11500 & 6.20 & & & & $0.0^{d}$ & & & 8 \\
\hline$\phi$ Her & 11781 & 5.65 & 6.01 & 6.12 & & 0.4 & 0.4 & {$[3]$} & 9.5 \\
\hline $56 \mathrm{Aqr}$ & 11977 & 5.25 & & & & $0.0^{d}$ & & & 25 \\
\hline HR 7694 A & 12000 & 6.70 & & & & $0.0^{d}$ & & & 8 \\
\hline HR 3302 & 12010 & 5.65 & & & & $0.0^{d}$ & & & 3 \\
\hline$v$ Her & 12013 & 6.40 & 6.02 & 6.10 & & 0.0 & 1.0 & [3] & 10 \\
\hline HR 7143 & 12077 & 5.65 & & & & 0.8 & 1.0 & [4] & 0.5 \\
\hline HR 7245 & 12193 & 5.35 & 5.33 & & & 0.5 & 1.0 & [3] & 0 \\
\hline 41 Eri B & 12250 & 5.75 & & & & $0.0^{d}$ & & & 12 \\
\hline AV Scl & 12400 & 7.00 & & & & 0.0 & & & 12 \\
\hline$\beta \mathrm{Scl}$ & 12476 & 6.60 & & 6.72 & & & 0.0 & [1] & 25 \\
\hline 41 Eri A & 12750 & 5.50 & & & & $0.0^{d}$ & & & 12 \\
\hline$\mu$ Lep & 12750 & 6.00 & 5.82 & 6.08 & & 1.6 & 1.6 & [3] & 16.5 \\
\hline $46 \mathrm{Aql}$ & 12914 & 4.95 & 4.97 & & & 0.3 & 0.6 & [3] & 0 \\
\hline 87 Psc & 13126 & 5.75 & & 6.09 & & & 1.5 & [1] & 21 \\
\hline HR 6520 & 13163 & 6.20 & & & 6.15 & 0.2 & & & 2.8 \\
\hline $112 \mathrm{Her}$ & 13294 & 6.10 & 6.34 & 6.07 & & 1.0 & 0.5 & [5] & 5 \\
\hline$\kappa \mathrm{Cnc}$ & 13470 & 5.80 & 6.76 & 5.78 & & 0.0 & 0.0 & [3] & 6.5 \\
\hline HR 7361 & 13570 & 5.50 & 5.62 & 5.61 & & & 0.7 & [3] & 8 \\
\hline HR 6759 & 13890 & 6.00 & & & & 0.0 & & & 2.5 \\
\hline$\theta$ Hyi & 14106 & 5.80 & & & & $0.0^{d}$ & & & 36 \\
\hline HR 4089 & 15126 & 5.15 & & & & $0.0^{d}$ & & & 7 \\
\hline
\end{tabular}

${ }^{a} \log N_{\mathrm{Hg}}$ as determined from the $\mathrm{Hg}$ II $\lambda 3984$ feature.

${ }^{b}$ Abundances from Woolf \& Lambert (1999a) reduced by 0.2 dex due to the different log $g f$ value adopted for the $\mathrm{Hg}$ II $\lambda 3984$ line.

${ }^{c} \xi_{\mathrm{t}}$ determined from comparison between the $\lambda 3984$ and $\lambda 6149$ line.

${ }^{d} \xi_{\mathrm{t}}$ assumed to be $0 \mathrm{~km} \mathrm{~s}^{-1}$, no $\xi_{\mathrm{t}}$ available.

References: [A] Woolf \& Lambert (1999a), [B] Smith (1997), [C] Hubrig et al. (1999b), [1] Jomaron et al. (1999), [2] Wahlgren et al. (1994), [3] Woolf \& Lambert (1999a), [4] Smith (1997), [5] Ryabchikova et al. (1996).

the radiative diffusion hypothesis developed by Michaud (1970) is the most commonly accepted theory for producing the observed anomalous elemental abundances and isotopic compositions. Michaud et al. (1974) suggested a scenario that would produce the observed abundance and isotopic anomalies of mercury in $\mathrm{HgMn}$ stars. Their basic assumptions are that the force of radiation exerted on the mercury atoms is substantially larger than the gravitational field in the region where mercury is singly ionized, and that the two forces are almost equal in the region where mercury is twice ionized. One of the possible results of this model would be that a cloud of mercury would form in the upper part of the HgMn star atmosphere at a location where the radiative and gravitational forces on $\mathrm{Hg}$ II are approximately equal, and where $N\left(\mathrm{Hg}_{\text {II }}\right)$ equals $N\left(\mathrm{Hg}_{\text {III }}\right)$. In this case the close balance between the two forces could allow the small mass differences of the isotopes to create the observed isotopic anomalies.

In the absence of convection zones and other mixing mechanisms it is very likely that the radiative diffusion hypothesis is responsible for many of the HgMn star abundance anomalies. However, this mechanism alone may not account for all observed abundance and isotopic anomalies. Modelling by 
Table 5. The isotope mixture of the $\mathrm{Hg}$ II $\lambda 3984$ line in $\mathrm{HgMn}$ stars.

\begin{tabular}{|c|c|c|c|c|c|c|c|c|}
\hline \multirow[t]{2}{*}{ Star } & \multirow{2}{*}{$\begin{array}{l}T_{\text {eff }} \\
(\mathrm{K})\end{array}$} & \multicolumn{7}{|c|}{ isotope mixture } \\
\hline & & 196 & 198 & 199 & 200 & 201 & 202 & 204 \\
\hline HD 124740 & 10350 & $0.0 \pm 0.2$ & $0.75 \pm 0.3$ & $0.75 \pm 0.3$ & $7.0 \pm 1.0$ & $4.0 \pm 0.5$ & $43.0 \pm 4.0$ & $44.5 \pm 4.0$ \\
\hline$v \mathrm{Cnc}$ & 10375 & $0.0 \pm 0.5$ & $0.0 \pm 0.5$ & $0.1 \pm 0.5$ & $0.5 \pm 1.0$ & $0.7 \pm 1.0$ & $35.0 \pm 10.0$ & $63.7 \pm 15.0$ \\
\hline$\chi$ Lupi & 10608 & $0.0 \pm 0.1$ & $0.0 \pm 0.1$ & $0.0 \pm 0.1$ & $0.0 \pm 0.1$ & $0.1 \pm 0.1$ & $0.7 \pm 0.2$ & $99.2 \pm 0.3$ \\
\hline$\phi$ Phe & 10612 & $0.0 \pm 0.3$ & $1.0 \pm 0.7$ & $1.0 \pm 0.7$ & $1.0 \pm 0.5$ & $1.0 \pm 0.5$ & $2.0 \pm 0.8$ & $94.0 \pm 5.0$ \\
\hline HR 7775 & 10750 & $0.0 \pm 0.1$ & $0.1 \pm 0.1$ & $0.0 \pm 0.1$ & $0.0 \pm 0.1$ & $0.4 \pm 0.2$ & $37.5 \pm 2.0$ & $62.0 \pm 3.0$ \\
\hline HR 4072 & 10900 & $0.0 \pm 0.1$ & $0.0 \pm 0.1$ & $0.1 \pm 0.1$ & $0.5 \pm 0.3$ & $0.7 \pm 0.4$ & $45.0 \pm 5.0$ & $53.7 \pm 5.0$ \\
\hline $28 \mathrm{Her}$ & 10908 & $0.0 \pm 0.2$ & $0.5 \pm 0.4$ & $0.5 \pm 0.4$ & $0.5 \pm 0.2$ & $0.5 \pm 0.4$ & $2.0 \pm 0.8$ & $96.0 \pm 3.0$ \\
\hline HR 4487 & 11020 & $0.0 \pm 0.5$ & $0.0 \pm 1.0$ & $0.0 \pm 1.0$ & $70.0 \pm 20.0$ & $10.0 \pm 8.0$ & $10.0 \pm 5.0$ & $10.0 \pm 5.0$ \\
\hline HR 1800 & 11088 & $0.0 \pm 0.1$ & $0.0 \pm 0.2$ & $0.5 \pm 0.2$ & $1.5 \pm 0.5$ & $3.0 \pm 1.5$ & $58.5 \pm 4.0$ & $36.5 \pm 3.0$ \\
\hline$\iota \mathrm{CrB}$ & 11250 & $0.0 \pm 0.1$ & $0.8 \pm 0.7$ & $0.8 \pm 0.6$ & $1.5 \pm 1.0$ & $2.5 \pm 1.2$ & $58.4 \pm 9.0$ & $36.0 \pm 5.0$ \\
\hline$\phi$ Her & 11781 & $0.0 \pm 0.3$ & $3.5 \pm 2.0$ & $2.5 \pm 1.5$ & $4.0 \pm 2.0$ & $2.0 \pm 3.0$ & $31.5 \pm 10.0$ & $56.5 \pm 8.0$ \\
\hline $56 \mathrm{Aqr}$ & 11977 & $0.15 \pm 0.2$ & $10.0 \pm 10.0$ & $16.9 \pm 15.0$ & $23.1 \pm 15.0$ & $13.2 \pm 20.0$ & $29.8 \pm 10.0$ & $6.85 \pm 10.0$ \\
\hline HR 3302 & 12010 & $0.1 \pm 0.1$ & $0.1 \pm 0.2$ & $0.1 \pm 0.2$ & $0.1 \pm 0.1$ & $0.1 \pm 0.3$ & $1.5 \pm 0.3$ & $98.0 \pm 1.5$ \\
\hline$v$ Her & 12013 & $0.0 \pm 0.2$ & $1.0 \pm 1.0$ & $1.5 \pm 1.0$ & $5.0 \pm 2.0$ & $0.5 \pm 1.0$ & $45.0 \pm 11.0$ & $47.0 \pm 7.0$ \\
\hline HR 7143 & 12077 & $0.0 \pm 0.1$ & $14.0 \pm 3.0$ & $5.0 \pm 2.0$ & $34.0 \pm 1.5$ & $8.0 \pm 2.0$ & $33.0 \pm 1.5$ & $6.0 \pm 0.5$ \\
\hline HR 7245 & 12193 & $0.0 \pm 0.1$ & $13.5 \pm 4.0$ & $12.0 \pm 3.5$ & $24.0 \pm 1.0$ & $1.5 \pm 0.5$ & $26.0 \pm 1.5$ & $23.0 \pm 1.0$ \\
\hline 41 Eri B & 12250 & $0.0 \pm 0.4$ & $5.0 \pm 5.0$ & $5.0 \pm 5.0$ & $10.0 \pm 7.0$ & $5.0 \pm 8.0$ & $47.0 \pm 18.0$ & $28.0 \pm 10.0$ \\
\hline AV Scl & 12400 & $0.0 \pm 0.3$ & $0.0 \pm 0.5$ & $0.8 \pm 1.0$ & $2.0 \pm 1.5$ & $2.0 \pm 2.0$ & $5.0 \pm 3.0$ & $90.2 \pm 10.0$ \\
\hline$\beta \mathrm{Scl}$ & 12476 & $0.0 \pm 0.5$ & $2.0 \pm 5.0$ & $2.0 \pm 5.0$ & $4.0 \pm 5.0$ & $4.0 \pm 8.0$ & $10.0 \pm 5.0$ & $78.0 \pm 20.0$ \\
\hline 41 Eri A & 12750 & $0.0 \pm 0.2$ & $0.0 \pm 0.3$ & $0.0 \pm 0.3$ & $0.0 \pm 0.5$ & $0.0 \pm 0.5$ & $3.0 \pm 5.0$ & $97.0 \pm 5.0$ \\
\hline$\mu$ Lep & 12750 & $0.0 \pm 0.4$ & $1.0 \pm 2.0$ & $6.0 \pm 5.0$ & $12.0 \pm 6.0$ & $15.0 \pm 10.0$ & $62.0 \pm 15.0$ & $4.0 \pm 10.0$ \\
\hline $46 \mathrm{Aql}$ & 12914 & $0.0 \pm 0.1$ & $0.0 \pm 0.1$ & $0.0 \pm 0.1$ & $0.0 \pm 0.2$ & $1.5 \pm 0.4$ & $2.5 \pm 0.2$ & $97.0 \pm 1.5$ \\
\hline 87 Psc & 13126 & $0.0 \pm 0.5$ & $6.0 \pm 10.0$ & $12.0 \pm 15.0$ & $25.0 \pm 15.0$ & $10.0 \pm 10.0$ & $37.0 \pm 20.0$ & $10.0 \pm 15.0$ \\
\hline HR 6520 & 13163 & $0.0 \pm 0.1$ & $10.0 \pm 4.0$ & $8.0 \pm 3.0$ & $58.5 \pm 7.0$ & $5.0 \pm 2.5$ & $18.0 \pm 3.0$ & $0.5 \pm 0.3$ \\
\hline 112 Her & 13294 & $0.0 \pm 0.2$ & $1.5 \pm 1.0$ & $1.5 \pm 1.0$ & $3.0 \pm 1.5$ & $3.0 \pm 2.5$ & $58.0 \pm 12.0$ & $33.0 \pm 6.0$ \\
\hline$\kappa \mathrm{Cnc}$ & 13470 & $0.0 \pm 0.3$ & $10.0 \pm 6.0$ & $10.0 \pm 6.0$ & $4.0 \pm 6.0$ & $30.0 \pm 12.0$ & $30.0 \pm 10.0$ & $16.0 \pm 5.0$ \\
\hline HR 7361 & 13570 & $0.15 \pm 0.3$ & $10.0 \pm 6.0$ & $16.9 \pm 8.0$ & $23.1 \pm 10.0$ & $13.2 \pm 7.0$ & $29.8 \pm 12.0$ & $6.85 \pm 5.0$ \\
\hline HR 6759 & 13890 & $0.0 \pm 0.1$ & $2.5 \pm 2.0$ & $4.0 \pm 3.0$ & $0.5 \pm 0.2$ & $0.5 \pm 0.3$ & $50.5 \pm 10.0$ & $42.0 \pm 6.0$ \\
\hline$\theta$ Hyi & 14106 & $0.15 \pm 0.5$ & $10.0 \pm 10.0$ & $16.9 \pm 20.0$ & $23.1 \pm 25.0$ & $13.2 \pm 15.0$ & $29.8 \pm 25.0$ & $6.85 \pm 10.0$ \\
\hline HR 4089 & 15126 & $0.0 \pm 0.5$ & $0.0 \pm 0.5$ & $0.0 \pm 1.0$ & $4.0 \pm 5.0$ & $8.0 \pm 5.0$ & $43.0 \pm 15.0$ & $45.0 \pm 10.0$ \\
\hline Terrest. & & 0.15 & 10.0 & 16.9 & 23.1 & 13.2 & 29.8 & 6.85 \\
\hline
\end{tabular}

Proffitt et al. (1999) show that radiative diffusion is unable to support the large abundance enhancements of mercury in the two HgMn stars $\chi$ Lupi and HR 7775. Furthermore, there is no simple way of utilizing the radiative diffusion mechanism to reproduce the isotopic mixture of mercury found in certain HgMn stars, such as HR 6759 (this study), 11 Per (Woolf \& Lambert 1999a), and HR 7245 (both studies). This suggests that there are other physical mechanism that contribute to the abundance and isotopic anomalies of mercury. Proffitt et al. suggest that the anomalous behaviour of mercury is the result of a combination of radiative diffusion, weak mixing currents, and mass loss. They also conclude that the mass-loss rate is an important factor in deciding the nature of the diffusion processes in these stars, and that non-LTE effects could create ionization anomalies for mercury.

One possible contributor to the isotopic and abundance anomalies is the light induce drift (LID) mechanism, first put forward by Atutuov \& Shalagin (1988). Nasyrov \& Shalagin (1993) suggest that LID, and not radiative diffusion, is responsible for the general abundance and isotopic anomalies in HgMn stars. The LID effect on a specific element is produced by there being an asymmetric light flux distribution across one of its spectral lines. This asymmetry is typically produced by the presence of a spectral line from another element in the line wing of the spectral line in question. The efficiency of LID is typically largest for atomic transitions involving low-excitation and high-excitation energy levels (i.e. the resonance lines). The position of the spectral line causing the asymmetric light flux distribution on the line profile of the other line decides whether the element will move upwards or downwards in the stellar atmosphere. This means that each element can be either enhanced, depleted or unaffected by LID depending on the random wavelength coincidence of spectral features. Thus if LID is the general cause of the $\mathrm{HgMn}$ star abundance anomalies, one would not expect to have a general enhancement of elements heavier than the iron-group, but rather randomly distributed enhanced, depleted and solar-like abundances. This abundance pattern is not supported by the observations of $\chi$ Lupi (Leckrone et al. 1999), for which the abundance of many post-iron group elements have been determined, or by observations of heavy elmental abundances in other HgMn stars. It may be possible that the extreme enhancements and depletions observed for specific elements and isotopes are caused by LID. Calculations by LeBlanc \& Michaud (1993) indicate that for helium the velocity introduced by LID is significantly higher than that caused by radiative 
acceleration, but they also conclude that the opposite is true for lithium, oxygen and mercury.

Recently, Aret \& Sapar (2002) suggested that LID is responsible for the isotopic anomalies of mercury in HgMn stars. It was further suggested that the isotopic composition would evolve over time from a terrestrial mixture towards one being purely ${ }^{204} \mathrm{Hg}$. If true, then inspection of the H-R diagram for HgMn stars would show trends with age of the isotopic composition, where a more terrestrial-like composition would lie at the hotter end of an isochrone. This simple scenario is not supported by analysis of HgMn star spectra. The colormagnitude diagram of Woolf \& Lambert (1999a) shows only random placement according to isotopic mixture. Likewise, isotopic information presented in the current study, when considered with the temperature-luminosity diagram of Adelman et al. (2003) for stars in common to both studies, does not show signs of isotopic mixture evolution. The components of the HgMn binaries 41 Eri (this paper) and 46 Dra (Woolf \& Lambert 1999a) have significantly different isotopic mixtures, yet atmospheric parameters that reflect similar mass and, therefore, evolutionary stages. The discovery of three young HgMn stars in the Orion OB1 association by Woolf \& Lambert (1999b) also provides constraints on the temporal development of spectral anomalies. Curiously, only two of these stars have developed a noticeable mercury enhancement, both of which appear to have isotope mixtures weighted towards the heavier isotopes. The evidence against the evolution of mercury isotopic mixture is convincing, yet provisional. The determination of both stellar luminosity and age have conciderable uncertainties or rely on stellar modelling and may influence our perception of the temporal development of the mercury isotopic composition.

Theoretical efforts have been made (e.g. Alecian 1993; Seaton 1996) that incorporate stellar age into diffusion calculations, with the conclusion that the $\mathrm{HgMn}$ star abundances should vary considerably during their main-sequence lifetimes. If the abundance anomalies change on time-scales much shorter than the main sequence phase of evolution, the observed star-to-star abundance differences could be explained by the age difference of the stars. This would mean that the current chemical composition of a HgMn star could be considered to represent a snapshot of time-variable processes occurring in the stellar atmosphere. Even if they are time dependant, the abundance and isotopic anomalies of a specific star are probably not only dictated by the stellar age. The fact that HgMn stars of similar age, as inferred from their atmospheric parameters, can have widely different abundances indicate that the occurrence of time-variable processes, if they exist, are complicated by other factors.

The explanation of the HgMn star phenomenon will prove to be the result of a complex interplay between a number of physical mechanisms. Possible hints of this are the claims of magnetic field and surface inhomogeniety detections in $\mathrm{HgMn}$ stars, both of which are quantities that could influence the HgMn star abundances. In addition, effects of stellar evolution, binarity, mass loss, and microturbulence could play important roles in the development of the observed anomalies. The large number of possible contributors means that further theoretical and observational studies must be undertaken before the $\mathrm{HgMn}$ star phenomenon can be fully understood.

Acknowledgements. We wish to thank the anonymous referee for useful comments.

\section{References}

Adelman, S. J., Adelman, A. S., \& Pintado, O. I. 2003, A\&A, 397, 267

Adelman, S. J., Gulliver, A. F., Kochukhov, O. P., \& Ryabchikova, T. A. 2002, ApJ, 575, 449

Alecian, G. 1993, Peculiar Versus Normal Phenomena in A-Type and Related Stars, ed. M. M. Dworetsky, F. Castelli, \& R. Faraggiana (San Fransisco: ASP), ASP Conf. Ser., 44, 450

Anders, E., \& Grevesse, N. 1989, Geochim. Cosmochim. Acta, 53, 197

Aret, A., \& Sapar, A. 2002, AN, 323, 21

Asplund, M., Nordlund, Å., Trampedach, R., \& Stein, R. F. 1999, A\&A, 346, L17

Atutov, S. N., \& Shalagin, A. M. 1988, Soviet Astron. Lett., 14, 284

Benck, E. C., Lawler, J. E., \& Dakin, J. T. 1989, JOSA B, 6, 11

Bidelman, W. P. 1962a, AJ, 67, 111

Bidelman, W. P. 1962b, S\&T, 23, 140

Brage, T., Proffitt, C. R., \& Leckrone, D. S. 1999, ApJ, 513, 524

Cowley, C. R., \& Aikman, G. C. L. 1975, PASP, 87, 513

Cowley, C. R. 1996, Model Atmospheres and Spectrum Synthesis, ed. S. J. Adelman, F. Kupka, \& W. W. Weiss, ASP Conf. Ser., 108, (San Fransisco: ASP), 223

Dolk, L., Wahlgren, G. M., Lundberg, H., et al. 2002, A\&A, 385, 111

Dworetsky, M. M. 1974, PASP, 86, 183

Dworetsky, M. M. 1980, A\&A, 84, 350

Guthrie, B. N. G. 1986, MNRAS, 220, 559

Hauck, B., \& Mermilliod, M. 1980, A\&AS, 40, 1

Heacox, W. D. 1979, ApJS, 41, 675

Hubrig, S., \& Mathys, G. 1995, Comm. Astrophys., 18, 167

Hubrig, S., Castelli, F., \& Wahlgren, G. M. 1999a, A\&A, 346, 139

Hubrig, S., Castelli, F., \& Mathys, G. 1999b, A\&A, 341, 190

Hubrig, S., Wahlgren, G. M., \& Dolk, L. 2003, in the poster paper Proc. of the 1st Potsdam Thinkshop on Sunspots and Starspots, ed. K. G. Strassmeier, \& A. Washuettl (Potsdam AIP), 109

Ilyin, I. 2000, Ph.D. Thesis, University of Oulu

Jomaron, C. M., Dworetsky, M. M., \& Allen, C. S. 1999, MNRAS, 303, 555

Kurucz, R. L. 1993, Synthesis Programs and Line Data Kurucz CD-ROM No. 18

Kurucz, R. L., \& Avrett E. H. 1981, Solar Spectrum Synthesis. I. A Sample Atlas from 224 to $300 \mathrm{~nm}$, Smithsonian Astrophys. Obs. Spec. Rept. 391

Landstreet, J. D. 1998, A\&A 338, 1041

Landstreet, J. D. 2003, in Modelling of Stellar Atmospheres, Proc. IAU Symp., 210, in press

LeBlanc, F., \& Michaud, G. 1993, ApJ, 408, 251

Leckrone, D. S. 1984, ApJ, 286, 725

Michaud, G. 1970, ApJ, 160, 641

Michaud, G., Reeves, H., \& Charland, Y. 1974, A\&A, 37, 313

Migdalek, J. 1976, Can. J. Phys., 54, 2272

Murakawa, K. 1932, Sci. Pap. Inst. Phys. Chem, Res, Tokyo, 18, 299

Napiwotski, R., Schoenberner, D., \& Wenske, N. 1993, A\&A, 268, $6 \mathrm{~T} 3$

Nasyrov, K. A., \& Shalagin, A. M. 1993, A\&A, 268, 201

Pinnington, E. H., Ansbacher, W, Kernahan, J. A., Ahmad, T., \& Ge, Z. Q. 1988, Can. J. Phys., 66, 960 
Proffitt, C. R., Brage, T., Leckrone, D. S., et al. 1999, ApJ, 512, 942

Reader, J., \& Sansonetti, C. J. 1986, Phys. Rev. A, 33, 1440

Ryabchikova, T. A., Zakharova, L. A., \& Adelman, S. J. 1996, MNRAS, 283, 1115

Sansonetti, C. J., \& Reader, J. 2001, Phys. Scr., 63, 219

Seaton, M. J. 1996, Ap\&SS, 237, 107

Sigut, T. A. A., Landstreet, J. D., \& Shorlin, S. L. S. 2000, ApJ, 530, L89

Smith, K. C. 1997, A\&A, 319, 928

Smith, K. C., \& Dworetsky, M. M. 1993, A\&A, 274, 335

Takada-Hidai, M., \& Jugaku, J. 1992, PASP, 104, 106

Tuominen, I. 1992, Nordic Optical Telescope News, No. 5, 15

Wahlgren, G. M., Adelman, S. J., \& Robinson, R. D. 1994, ApJ, 434, 349
Wahlgren, G. M., Leckrone, D. S., Johansson, S., Rosberg, M., \& Brage, T. 1995, ApJ, 444, 438

Wahlgren, G. M., Dolk, L., Kalus, G., Johansson, S., \& Litzén, U. 2000, ApJ, 539, 908

Wahlgren, G. M., \& Hubrig, S. 2000, A\&A, 362, L13

Wahlgren, G. M., Ilyin, I., \& Kochukhov, O. 2001, BAAS, 33, 1506

Wahlgren, G. M., Ilyin, I., Kochukhov, O., \& Hubrig, S. 2003, in the poster paper proceedings of the 1st Potsdam Thinkshop on Sunspots and Starspots, ed. K. G. Strassmeier, \& A. Washuettl (Potsdam: AIP), 87

White, R. E., Vaughan, A. H. Jr., Preston, G. W., \& Swings, J. P. 1976, ApJ, 204, 131

Woolf, V. M., \& Lambert, D. L. 1999a, ApJ, 521, 414

Woolf, V. M., \& Lambert, D. L. 1999b, ApJ, 520, L55 